\title{
Onsager Transport Coefficients and Transference Numbers in Polyelectrolyte Solutions and Polymerized Ionic Liquids
}

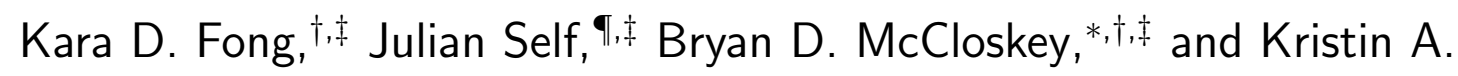 \\ Persson $* \boldsymbol{\Phi}, \ddagger$ \\ $\dagger$ Department of Chemical and Biomolecular Engineering, University of California, Berkeley \\ $\ddagger$ Energy Technologies Area, Lawrence Berkeley National Laboratory \\ \Department of Materials Science and Engineering, University of California, Berkeley \\ E-mail: bmcclosk@berkeley.edu; kapersson@lbl.gov
}

\begin{abstract}
Electrolytes featuring negatively-charged polymers such as nonaqueous polyelectrolyte solutions and polymerized ionic liquids are currently under investigation as potential high cation transference number $\left(t_{+}\right)$electrolytes for lithium ion batteries. Herein, we use coarse-grained molecular dynamics simulations to characterize the Onsager transport coefficients of polyelectrolyte solutions as a function of chain length and concentration. For all systems studied, we find that the rigorously computed transference number is substantially lower than that approximated by the ideal solution (Nernst-Einstein) equations typically used to characterize these systems due to the presence of strong anion-anion and cation-anion correlations. None of the polyelectrolyte solutions achieve $t_{+}$greater than that of the conventional binary salt electrolyte, with some solutions having negative $t_{+}$. This work demonstrates that the Nernst-Einstein assumption does not provide a physically meaningful estimate of the
\end{abstract}


transference number in these solutions and calls into question the expectation of polyelectrolytes to exhibit high cation transference number.

\section{Introduction}

High cation transference number $\left(t_{+}\right)$electrolytes, in which the majority of the electrolyte conductivity is attributed to the cation rather than the anion, have the potential to improve the power density and attainable state of charge of lithium ion batteries (LIBs). ${ }^{1-3} \mathrm{~A}$ common strategy in increasing $t_{+}$is to covalently append the electrolyte anion to the backbone of a polymer, thereby immobilizing the anion to yield a single-ion conductor. ${ }^{4,5}$ Maintaining acceptable conductivity in a dry (neat) single-ion conducting polymer, however, has proved challenging due to incomplete ion dissociation and/or low ion mobility. ${ }^{3,6,7}$

Polyelectrolyte solutions in which a lithium-neutralized polyanion is dissolved in a nonaqueous solvent have recently been proposed as alternatives to conventional solid-state singleion conductors. ${ }^{8-14}$ As these polyelectrolytes are entirely in the liquid phase, they could serve as a means to increase transference number without drastic conductivity losses and be directly used in existing cell designs. Indeed, initial studies have reported transference numbers greater than $0.8\left(t_{+} \sim 0.4 \text { for conventional LIB electrolytes }\right)^{15}$ with conductivities on the order of $0.1-1 \mathrm{mS} / \mathrm{cm}^{8,10,11,14}$ As the transference number is challenging to measure experimentally, ${ }^{3}$ the reported $t_{+}$values in these works are typically estimated by assuming that the solution behaves ideally, i.e., there are no correlations between ions in the electrolyte. This is referred to as the Nernst-Einstein (NE) approximation and yields an expression for transference number in terms of the self-diffusion coefficients of the cation and anion, $D_{+}$ and $D_{-}$, respectively:

$$
t_{+}^{\mathrm{NE}}=\frac{D_{+}}{D_{+}+D_{-}}
$$

While these experimental results seem promising, our previous work ${ }^{16}$ on one polyelectrolyte system, poly(allyl glycidyl ether-lithium sulfonate) (PAGELS) in dimethyl sulfoxide 
(DMSO), found that $t_{+}^{\mathrm{NE}}$ drastically overestimates the true transference number $\left(t_{+}^{\mathrm{NE}} \gg t_{+}\right)$. Using atomistic molecular dynamics simulations, it was shown that the anion-anion correlations inherently present between monomers on the same chain make a substantial contribution to the overall conductivity and therefore decrease the transference number relative to the ideal case.

It remains to be seen, however, whether the failure of Eq. (1) generalizes beyond the PAGELS system, and whether we can make any more general claims about the extent of non-idealities (ion correlations) in polyelectrolytes which hold independent of the electrolyte chemistry. Furthermore, we wish to understand (i) the emergence of polyelectrolytes' unique transport properties as we transition from a conventional monomeric electrolyte to oligo- and polyanions and (ii) how transport properties change as a function of concentration, including the extreme limit of solvent-free systems or polymerized ionic liquids, which are also under investigation as LIB electrolyte alternatives. ${ }^{17-19}$ While existing polyelectrolyte studies have extensively explored the polyion transport properties in these solutions, ${ }^{20,21}$ the behavior of the counterion and thus the battery-relevant transport properties of polyelectrolytes such as $t_{+}$are largely unexplored.

Herein, we use coarse-grained molecular dynamics simulations with explicit solvent to systematically study transport as a function chain length and concentration for a generic polyelectrolyte solution. We begin with an overview of the theoretical framework used to study electrolyte transport. This theory is based on the formulation of transport coefficients, denoted $L^{i j}$, which provide insight into the ion correlations between species in solution. We then present the transport coefficients computed for the coarse-grained polyelectrolyte model, providing a direct comparison between a conventional binary electrolyte and the polyelectrolyte systems. Next, we use the transport coefficients to calculate the transference number for these solutions, rationalizing the resulting trends based on ion correlations and ion pairing behavior. We find that the strong ion correlations in polyelectrolyte solutions generally yield conductivity and transference number values lower than in monomeric electrolytes, 
suggesting that - from a transport perspective - polyelectrolytes may not be a promising alternative to conventional battery electrolytes.

\section{Theory}

Herein, we analyze transport in electrolytes using the Onsager transport coefficients. The theoretical framework required to define these transport equations is derived and discussed in detail in Fong et al. ${ }^{22}$ Here we summarize only the main components of the theory. The reader may be more familiar with the Stefan-Maxwell equations for multicomponent diffusion, and by extension Newman's concentrated electrolyte theory, ${ }^{2}$ rather than the Onsager equations presented in this work. As discussed in Fong et al., ${ }^{22}$ both frameworks are thermodynamically consistent, and it is possible to map between the transport coefficients from the two approaches. ${ }^{22,23}$ However, the Onsager transport coefficients $L^{i j}$ have a more direct physical interpretation in terms of ion correlations, and only $L^{i j}$ may be computed directly from molecular simulations using Green-Kubo relations. Furthermore, while it is possible to obtain experimental quantities from the Stefan-Maxwell coefficients $K^{i j}$, the expressions for doing so are more complex than with $L^{i j}$, especially for systems with more than two ionic species. These advantages make the Onsager transport equations a clear choice for the analysis in this work. Nevertheless, we provide the Stefan-Maxwell coefficients $K^{i j}$ for the polyelectrolyte solutions studied herein in the SI for comparison (Figure S6).

The theory of nonequilibrium thermodynamics applied to electrolytes yields the following set of Onsager transport equations:

$$
c_{i}\left(\boldsymbol{v}_{i}-\boldsymbol{v}\right)=-\sum_{j} L^{i j} \nabla \bar{\mu}_{j}
$$

These equations relate the flux of species $i \boldsymbol{J}_{i}=c_{i}\left(\boldsymbol{v}_{i}-\boldsymbol{v}\right)$ to the thermodynamic driving force in the system: gradients in the electrochemical potential of each species $j, \bar{\mu}_{j}$. In Eq. (2), $c_{i}$ and $\boldsymbol{v}_{i}$ are the concentration and velocity of species $i$, respectively, and $\boldsymbol{v}$ is the mass-averaged 
velocity of the entire system. The constants of proportionality in this linear relationship are the transport coefficients $L^{i j}$. The transport matrix is symmetric by the Onsager reciprocal relations $\left(L^{i j}=L^{j i}\right)$ and subject to the constraint that $\sum_{i} M_{i} L^{i j}=0$, where $M_{i}$ is the molecular weight of species $i$. The latter relation is a consequence of the fact that all mass fluxes must sum to zero, i.e., $\sum_{i} M_{i} \boldsymbol{J}_{i}=0$. Based on these two constraints, an electrolyte of $n$ components has $n(n-1) / 2$ independent transport coefficients. The polyelectrolyte solutions studied herein have three components (polyanion, cation, and solvent), yielding three independent transport coefficients: $L^{++}, L^{--}$, and $L^{+-}$.

On a molecular level, $L^{i j}$ may be interpreted as giving the degree of correlation between the motion of species $i$ and $j$. This is apparent quantitatively from the Green-Kubo relations for $L^{i j}$, where the correlation function in the fluxes of species $i$ and $j$ gives the transport coefficients directly from molecular simulations:

$$
L^{i j}=\frac{V}{3 k_{\mathrm{B}} T} \int_{0}^{\infty} d t\left\langle\boldsymbol{J}_{i}(t) \cdot \boldsymbol{J}_{j}(0)\right\rangle
$$

where $V$ is volume and $k_{\mathrm{B}} T$ is the thermal energy. Note that the overall flux of species $i$, $\boldsymbol{J}_{i}=c_{i}\left(\boldsymbol{v}_{i}-\boldsymbol{v}\right)$, is based on the average motion of all particles of species $i$. The quantity $\boldsymbol{v}_{i}$ can be expressed as $\boldsymbol{v}_{i}=\frac{1}{N_{i}} \sum_{\alpha} \boldsymbol{v}_{i}^{\alpha}$, where the index $\alpha$ refers to an individual particle of type $i$ and $N_{i}$ is the number of particles of type $i$ in the system.

The Green-Kubo relations of Eq. (3) may be equivalently written in terms of particle positions, rather than velocities. This form of the equation, which is analogous to computing self-diffusion coefficients from the mean-squared displacement of particle positions, is the one used to compute $L^{i j}$ in this work:

$$
L^{i j}=\frac{1}{6 k_{\mathrm{B}} T V} \lim _{t \rightarrow \infty} \frac{d}{d t}\left\langle\sum_{\alpha}\left[\boldsymbol{r}_{i}^{\alpha}(t)-\boldsymbol{r}_{i}^{\alpha}(0)\right] \cdot \sum_{\beta}\left[\boldsymbol{r}_{j}^{\beta}(t)-\boldsymbol{r}_{j}^{\beta}(0)\right]\right\rangle,
$$

where $\boldsymbol{r}_{i}^{\alpha}$ is the position of particle $\alpha$ relative to the center-of-mass position of the entire system. 
Let us further consider the diagonal terms of the transport matrix, $L^{i i}$ :

$$
L^{i i}=\frac{1}{6 k_{\mathrm{B}} T V} \lim _{t \rightarrow \infty} \frac{d}{d t} \sum_{\alpha} \sum_{\beta}\left\langle\left[\boldsymbol{r}_{i}^{\alpha}(t)-\boldsymbol{r}_{i}^{\alpha}(0)\right] \cdot\left[\boldsymbol{r}_{i}^{\beta}(t)-\boldsymbol{r}_{i}^{\beta}(0)\right]\right\rangle
$$

These transport coefficients are composed of two contributions: when $\alpha=\beta$, we take the autocorrelation function of the flux of particle $\alpha$, and when $\alpha \neq \beta$, we compute the crosscorrelations between two distinct particles. These two contributions may be denoted as self and distinct terms, respectively:

$$
\begin{gathered}
L_{\mathrm{self}}^{i i}=\frac{1}{6 k_{\mathrm{B}} T V} \lim _{t \rightarrow \infty} \frac{d}{d t} \sum_{\alpha}\left\langle\left[\boldsymbol{r}_{i}^{\alpha}(t)-\boldsymbol{r}_{i}^{\alpha}(0)\right]^{2}\right\rangle \\
L_{\text {distinct }}^{i i}=\frac{1}{6 k_{\mathrm{B}} T V} \lim _{t \rightarrow \infty} \frac{d}{d t} \sum_{\alpha} \sum_{\beta \neq \alpha}\left\langle\left[\boldsymbol{r}_{i}^{\alpha}(t)-\boldsymbol{r}_{i}^{\alpha}(0)\right] \cdot\left[\boldsymbol{r}_{i}^{\beta}(t)-\boldsymbol{r}_{i}^{\beta}(0)\right]\right\rangle .
\end{gathered}
$$

Note that $L^{i i}=L_{\text {self }}^{i i}+L_{\text {distinct }}^{i i}$. The distinct terms capture correlations between particles (non-idealities) while the self terms may be considered ideal or Nernst-Einstein contributions to transport. The latter may be directly related to the self-diffusion coefficient by

$$
L_{\mathrm{self}}^{i i}=\frac{D_{i} c_{i}}{k_{\mathrm{B}} T}
$$

The second law of thermodynamics imposes some restrictions on the possible values of each transport coefficient. While the distinct terms may take on positive or negative values, the self terms and the overall $L^{++}$and $L^{--}$must always be positive. The physical interpretation of each $L^{i j}$ will be discussed in more detail in the Results and Discussion.

The transport coefficients may be combined to yield experimentally-relevant transport properties, namely the ionic conductivity $\kappa$, the electrophoretic mobility $u_{i}$, and the transference number $t_{i}$ :

$$
\kappa=F^{2} \sum_{i} \sum_{j} L^{i j} z_{i} z_{j}
$$




$$
u_{i}=\sum_{j} L^{i j} \frac{z_{j} F}{c_{i}}
$$

and

$$
t_{i}=\frac{F z_{i} c_{i} u_{i}}{\kappa}=\frac{\sum_{j} L^{i j} z_{i} z_{j}}{\sum_{k} \sum_{l} L^{k l} z_{k} z_{l}}
$$

where $F$ is Faraday's constant and $z_{i}$ is the charge valence of species $i$.

The ideal solution transference number, $t_{i}^{\mathrm{NE}}$, can be computed analogously by assuming there are no ion correlations, thereby eliminating the off-diagonal components of the transport matrix $\left(L^{+-}\right)$as well as the distinct terms. This yields an expression in terms of only the self transport coefficients:

$$
t_{i}^{\mathrm{NE}}=\frac{z_{i}^{2} L_{\mathrm{self}}^{i i}}{\sum_{j} z_{j}^{2} L_{\mathrm{self}}^{j j}}
$$

For the cation transference number of a binary electrolyte, incorporating Eq. (8) and the condition of electroneutrality, $\sum_{i} z_{i} c_{i}=0$, yields

$$
t_{+}^{\mathrm{NE}}=\frac{z_{+} D_{+}}{z_{+} D_{+}-z_{-} D_{-}}
$$

When computing transport coefficients for a polyelectrolyte system, we may treat either individual monomers or polymer chains as a whole as the anionic species. In the analysis in this work, we choose the former. While this will necessarily impact the transport coefficients, experimentally-measurable properties such as the conductivity, mobility, and transference number will be equivalent with either approach (see the SI for a more detailed discussion). In Eq. (1), it has implicitly been assumed that $z_{-}=-1$, i.e., that each monomer is considered to be an independent anionic species. 


\section{Results and Discussion}

\section{Onsager transport coefficients}

Here we present the computed the Onsager transport coefficients for polyelectrolyte solutions using a coarse-grained molecular dynamics model based on the classical Kremer-Grest bead-spring chain ${ }^{24,25}$ with explicit solvent. We simulate chain lengths ranging from 1 to 25, noting that based on previous studies using similar polymer models we do not expect significant entanglement effects for these chain lengths. ${ }^{24,26,27}$ These polyelectrolytes are studied at four concentrations ranging from $0.001 \sigma^{-3}$ to $0.05 \sigma^{-3}$, where $\sigma$ is the Lennard-Jones unit of distance and the diameter of each particle in the system. Mapping the size of one monomer bead to that of a polystyrene sulfonate monomer $(2.5 \AA)$, this concentration range approximately corresponds to 0.1 to $5 \mathrm{M}$ (see the Methods section for a more detailed discussion).

In this section, we discuss the transport coefficients in these solutions and show how they may be interpreted to gain physical insight into transport phenomena in polyelectrolytes. Each of the transport coefficients $L^{i j}$ quantifies a different aspect of ion motion or ion-ion correlation, illustrated qualitatively in Figure 1a. As a representative example, let us analyze $L^{i j}$ as a function of chain length at a concentration of $0.01 \sigma^{-3}$, given in Figure $1 \mathrm{~b}$ with the diagonal terms $L^{i i}$ split into self and distinct components. The other concentrations studied give similar trends, as shown in Figures S1 and S2.

The two self terms $\left(L_{\text {self }}^{++}\right.$and $\left.L_{\text {self }}^{--}\right)$given in Figure $1 \mathrm{~b}$ are proportional to the selfdiffusion coefficients (Eq. (8); diffusion coefficient data are given directly in Figure S8). For the monomeric systems $(N=1)$ corresponding to conventional binary electrolytes, $L_{\text {self }}^{++}$is necessarily equal to $L_{\text {self }}^{--}$, as the cation and anion in the model are equivalent in their mass, size, and excluded volume (Lennard-Jones) interactions. As $N$ increases, both $L_{\text {self }}^{++}$and $L_{\text {self }}^{--}$decrease, a trend which aligns with the intuitive expectation of diffusion coefficients to decrease as the chain becomes larger and the overall solution more viscous. The decrease in 

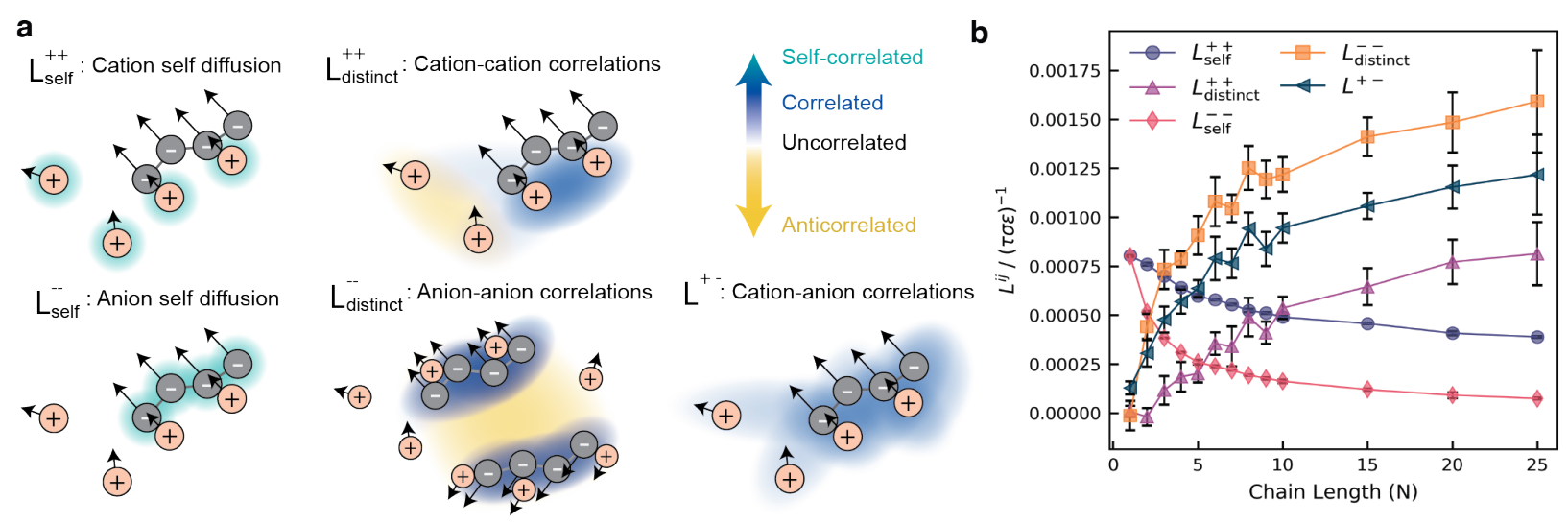

Figure 1: Transport coefficients in polyelectrolyte solutions. (a) Schematics illustrating the ion correlations captured by each transport coefficient. The arrows on each ion indicate direction of motion, and the shaded regions give qualitative information on the correlation between groups of ions according to the colorbar in the upper right. Note that solvent molecules are modeled explicitly but are omitted from the schematic for clarity. (b) Each $L^{i j}$ as a function of chain length at a concentration of $0.01 \sigma^{-3}$, split into self and distinct contributions.

$L_{\text {self }}^{--}$is more pronounced due to the bulky nature of the polymer chain.

The $L^{+-}$transport coefficient captures correlations between cations and anions. The value of $L^{+-}$is much lower than that of either $L^{++}$or $L^{--}$for the $N=1$ system but becomes increasingly significant as chain length increases. This trend suggests an increased prevalence of ion pairing at higher $N$, as expected from theories for counterion condensation in polyelectrolytes ${ }^{28}$ and confirmed directly in these simulations vide infra. Note that when computing ionic conductivity via Eq. (9), the $L^{+-}$term will be subtracted from the total value. Thus, as expected, cation-anion correlations decrease the overall conductivity.

Let us now turn to the distinct terms, $L_{\text {distinct }}^{++}$and $L_{\text {distinct }}^{--}$, which describe cation-cation and anion-anion correlations, respectively. In general, one would expect distinct ions of the same species to interact very little, in which case the distinct terms would be approximately zero, or to repel, leading to negative values of the distinct terms reflecting anti-correlated motion. Indeed, the monomeric $(N=1)$ systems studied here show very small contributions of $L_{\text {distinct }}^{++}$and $L_{\text {distinct }}^{--}$, with the values at high concentration being slightly negative (Figure S2d). As chain length increases, however, we deviate from this expected behavior and observe 
that $L_{\text {distinct }}^{++}$and $L_{\text {distinct }}^{--}$increase with chain length. These very positive values suggest highly correlated motion, i.e. groups of like-charge ions moving together for appreciable periods of time. Like the trend in $L^{+-}$, this behavior can be rationalized by increased ion aggregation. The increase of $L_{\text {distinct }}^{++}$with chain length reflects the presence of multiple cations bound to the same chain, which will move together for the lifetime of the ion aggregate. As chain length increases (and with it the extent ion pairing), we expect more cations on average to be bound to a given chain, resulting in an increase in the correlations between distinct cations. The increase in $L_{\text {distinct }}^{--}$with chain length is even more pronounced: for most chain lengths, this term dominates over all other transport coefficients and will thus strongly impact conductivity and transference number. The total value of $L_{\text {distinct }}^{--}$contains contributions from correlations between anions tethered to the same chain as well as anions on different chains. The former will generate a positive contribution to $L_{\text {distinct }}^{--}$, as anions on a given chain are constrained to move together and are thus highly correlated, while the latter will give a negative contribution, as two negatively-charged macroions will repel each other and move in an anti-correlated manner. In all systems studied, we observe that intra-chain correlations dominate over inter-chain correlations (Figure S5), yielding the strong positive trend in $L_{\text {distinct }}^{--}$with chain length.

\section{Transference numbers}

Having computed the transport coefficients for a range of polyelectrolyte solutions, we may now combine these $L^{i j}$ to obtain experimentally relevant quantities. Of particular interest is the cation transference number, shown in Figure 2a. We observe that for all concentrations studied, the transference number decreases as chain length increases, with the monomeric electrolyte having the highest transference number. Given that ionic conductivity generally decreases as chain length increases (Figure S7), these results suggest that the polyelectrolyte solutions studied herein would not yield any advantages in battery performance relative to a conventional monomeric electrolyte. 

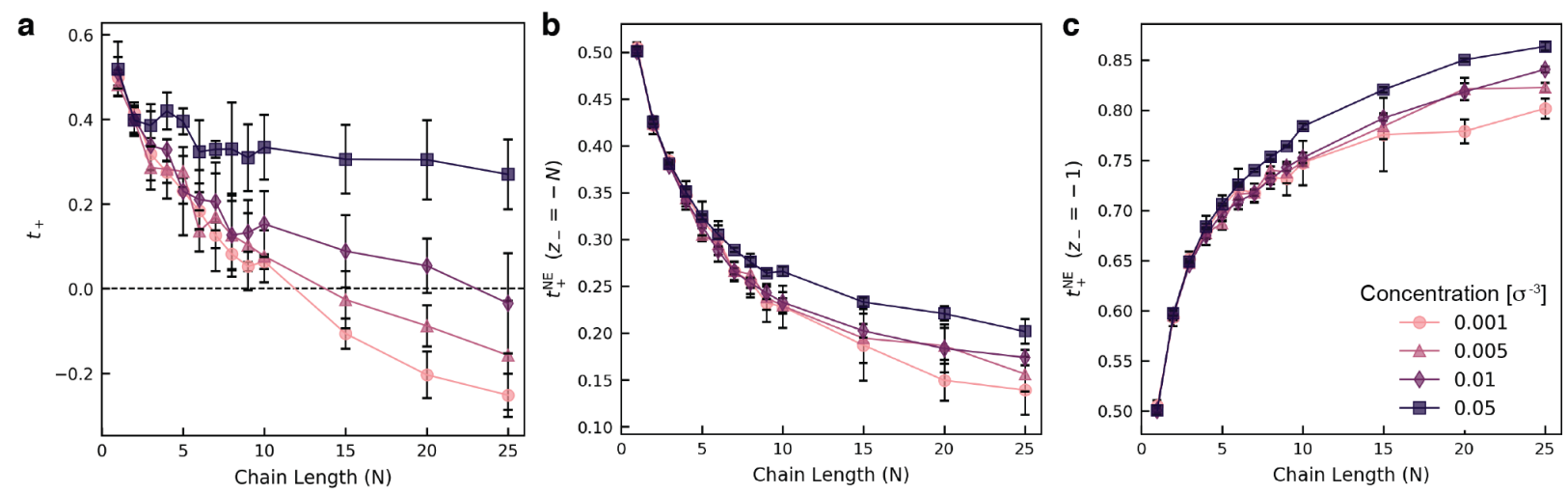

$t_{+}=\frac{L^{++}-L^{+-}}{L^{++}-2 L^{+-}+L^{--}}$

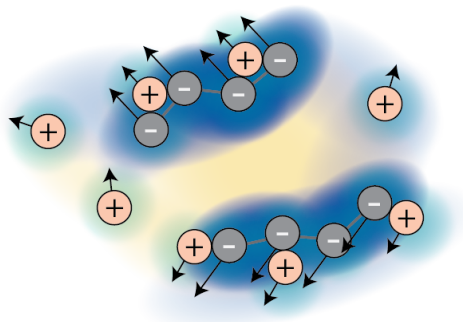

All correlations accounted for

$$
t_{+}^{\mathrm{NE}}\left(z_{-}=-N\right)=\frac{D_{+}}{D_{+}+N D_{-}}
$$

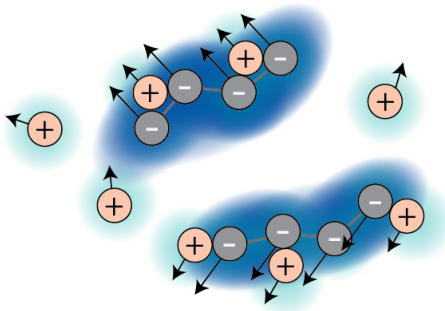

Self- and intra-chain correlations only

$$
t_{+}^{\mathrm{NE}}\left(z_{-}=-1\right)=\frac{D_{+}}{D_{+}+D_{-}}
$$

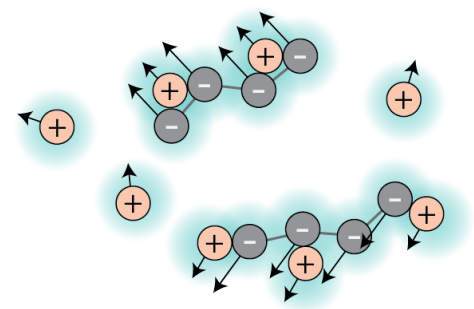

Self-correlations only

Figure 2: Cation transference number as a function of chain length. (a) Rigorously computed $t_{+}$obtained from the Onsager transport coefficients and accounting for all ion correlations present in solution. (b) and (c) $t_{+}^{\mathrm{NE}}$, the ideal solution transference number ignoring correlations between ions. These are written in terms of the self-diffusion coefficients, rather than Onsager transport coefficients, using Eq. (8). (b) Treating entire polymer chains as the anionic species $\left(z_{-}=-N\right)$ accounts for intra-chain correlations but ignores correlations between chains and between cations and anions. (c) Treating individual monomers as the anionic species $\left(z_{-}=-1\right)$ ignores all ionic correlations. Ion correlations are depicted according to the colormap in Figure 1.

The values of the rigorously computed $t_{+}$stand in stark contrast with the ideal solution transference number, $t_{+}^{\mathrm{NE}}$. Recall from Eq. (13) that there are two options for computing $t_{+}^{\mathrm{NE}}$ : we may choose to treat each monomer as a separate ion, in which case the $z_{-}$appearing in Eq. (13) is -1 , or we may treat the entire polymer chain as the anionic species, in which case $z_{-}=-N$. The resulting $t_{+}^{\mathrm{NE}}$ values based on each of these choices are given in Figures $2 \mathrm{~b}$ and $\mathrm{c}$. We observe that $t_{+}^{\mathrm{NE}}\left(z_{-}=-N\right)$ qualitatively reproduces the trend that transference number decreases as chain length increases, but in most cases it overestimates the rigorously computed $t_{+}$. This equation ignores correlations between different chains and 
between cations and anions, but it captures intra-chain correlations by treating the entire chain as a single unit. In contrast, $t_{+}^{\mathrm{NE}}\left(z_{-}=-1\right)$ does not account for any correlations between ions, capturing only self-diffusion. This assumes that monomers on the same chain move completely independently, which is obviously incorrect given the fact that they are covalently bonded. The resulting values for $t_{+}^{\mathrm{NE}}\left(z_{-}=-1\right)$ in Figure $2 \mathrm{c}$ increase with chain length, reaching values as high as 0.86 . This approximation drastically overestimates the true $t_{+}$and clearly does not provide a physically meaningful estimate of transference number in polyelectrolytes. In experimental systems where the transference number cannot be rigorously measured and only self-diffusion coefficients are available, $t_{+}^{\mathrm{NE}}\left(z_{-}=-N\right)$ provides a much more reasonable estimate of $t_{+}$.

We now seek to rationalize the transference number trends in Figure 2 based on the ion correlations in the electrolyte. While each of the $L^{i j}$ affect the observed trends in the transference number, the general decrease in $t_{+}$with increasing chain length can be most directly understood through the trends in $L_{\text {self }}^{--}$and $L_{\text {distinct }}^{--}$, which are shown in Figure 3a. The data in this figure are divided by the total concentration to yield the contribution to each transport coefficient per ion; each of the other transport coefficients is plotted in this manner in Figure S3. For each concentration, the $L_{\text {self }}^{--}$term (and thus the self-diffusion coefficient) decreases with $\mathrm{N}$. Indeed, the fact that a long polymer chain moves more slowly than a monomer is the basis for the intuitive notion that the anion in a polyelectrolyte solution should carry less current than the cation to yield a high $t_{+}$. As Eq. (13) for $t_{+}^{\mathrm{NE}}$ includes only the self terms (assuming all distinct terms to be zero), it is clear why $t_{+}^{\mathrm{NE}}\left(z_{-}=-1\right)$ in Figure 2c exhibits such high values. In polyelectrolyte systems, however, it is unreasonable to ignore the distinct terms when estimating $t_{+}$, as we observe that the magnitude of $L_{\text {distinct }}^{--}$ surpasses that of $L_{\text {self }}^{--}$for all but the shortest chain lengths. As mentioned above, this increase in $L_{\text {distinct }}^{--}$is due to the fact that increasing $N$ leads to more anions being covalently bound and thus constrained to move together, a phenomena which will be general to any polyelectrolyte regardless of the chemical properties of the polymer or solvent. The sum 
of the self and distinct terms, i.e., the overall $L^{--}$, increases with respect to chain length (Figure S1), corresponding to a decreasing cation transference number.
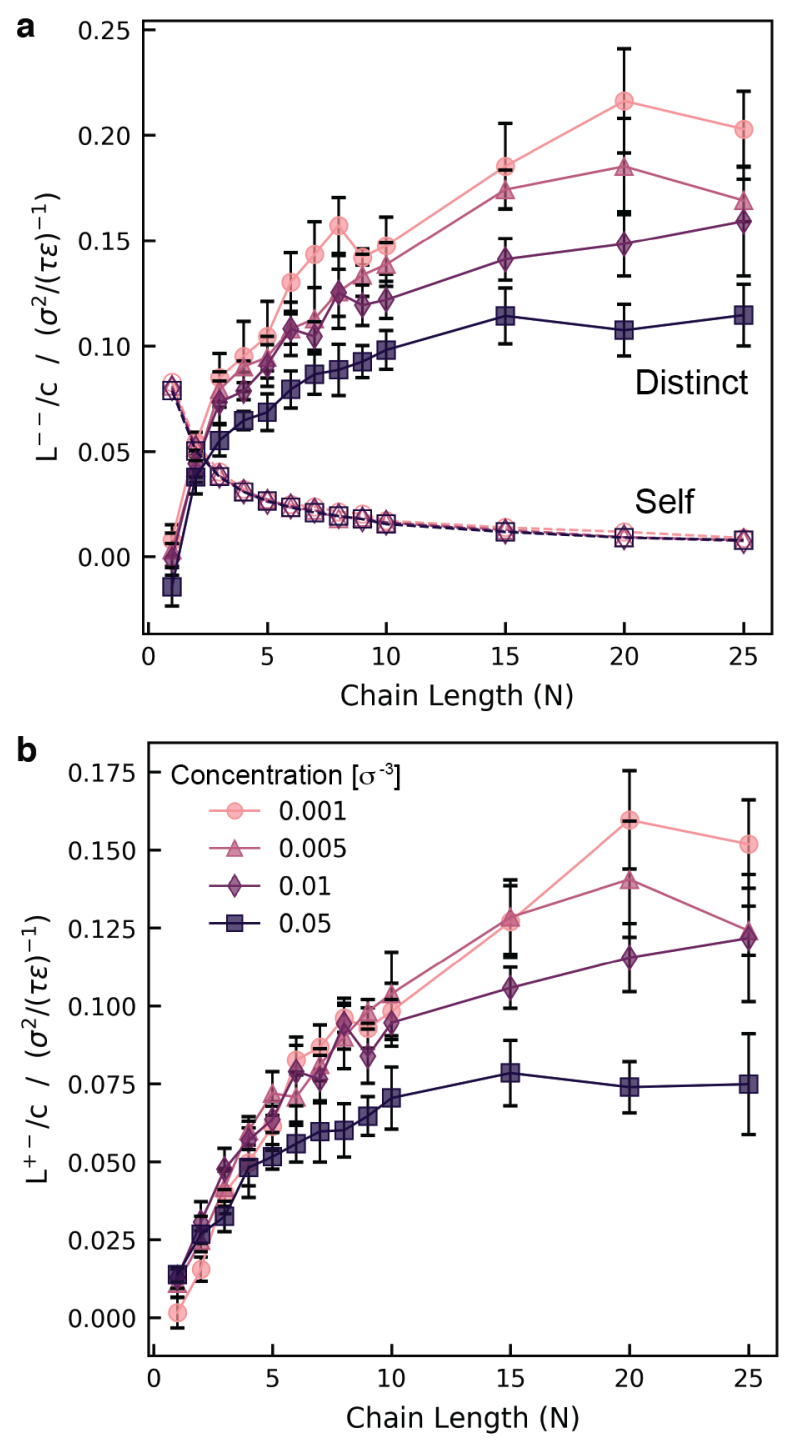

Figure 3: Comparison of anion transport coefficients for each concentration and chain length. (a) $L_{\text {self }}^{--}$(dashed lines) and $L_{\text {distinct }}^{--}$(solid lines), all divided by concentration to give the contributions to each transport coefficient per ion. (b) $L^{+-}$divided by concentration.

In addition to the trend with respect to chain length, we also note that $t_{+}$increases with concentration, similarly to previous results obtained with all-atom simulations on PAGELS in DMSO. ${ }^{16}$ This can be most clearly understood in terms of cation-anion correlations, quantified in Figure 3b. The contribution to $L^{+-}$per ion decreases as the concentration increases, 
yielding higher $t_{+}$for more concentrated solutions. In order to rationalize the trend of decreasing cation-anion correlations as concentration increases, let us consider the ion pairing behavior of the solutions. Ion pairing is conventionally defined based on a structural analysis of the solution: a cation is considered to be paired at a given time if it is within some specific distance of an anion. The fraction of cations in ion pairs or larger aggregates based on this type of analysis is given in Figure 4a. The resulting trend that ion pairing increases with concentration, however, is not consistent with the trend of decreasing $L^{+-} / c$ with concentration, suggesting that this static structural analysis does not provide a meaningful assessment of ion correlations in the system. Instead, the trends in $L^{+-} / c$ must be understood with a dynamic analysis of ion pairing, shown in Figure 4b. Here we quantify the ion pair residence time, or the characteristic time for an ion pair to exist before breaking apart. ${ }^{29-31}$ Despite the fact that more ions are paired at high concentrations, each of these pairs has a shorter lifetime, resulting in overall less correlated cation-anion motion and higher cation transference number. This phenomenon is illustrated schematically in Figures 4c and d. The negative correlation between static ion pairing fraction and dynamic ion pair lifetime was similarly observed in MD simulations of PAGELS in DMSO, ${ }^{16}$ suggesting that this may be a common phenomenon in polyelectrolyte solutions.

The lowest concentrations studied yield negative $t_{+}$at high chain lengths. This phenomenon corresponds to the presence of negatively charged aggregates which contribute substantially to the conductivity. For example, if a single cation is bound to a polyanion chain to yield an aggregate with large negative charge, upon application of an electric field that bound cation will move along with the polymer towards higher electric potential (in the "wrong direction"). As these negatively-charged aggregates are a natural occurrence in polyelectrolytes, it is unsurprising that we observe $t_{+}<0$ (or equivalently $t_{-}>1$ ) for cases at low concentration when ion pairs are long-lived. In fact, solutions with $t_{-}>1$ have been widely reported experimentally for dilute, aqueous polyelectrolytes. ${ }^{32-36}$ 


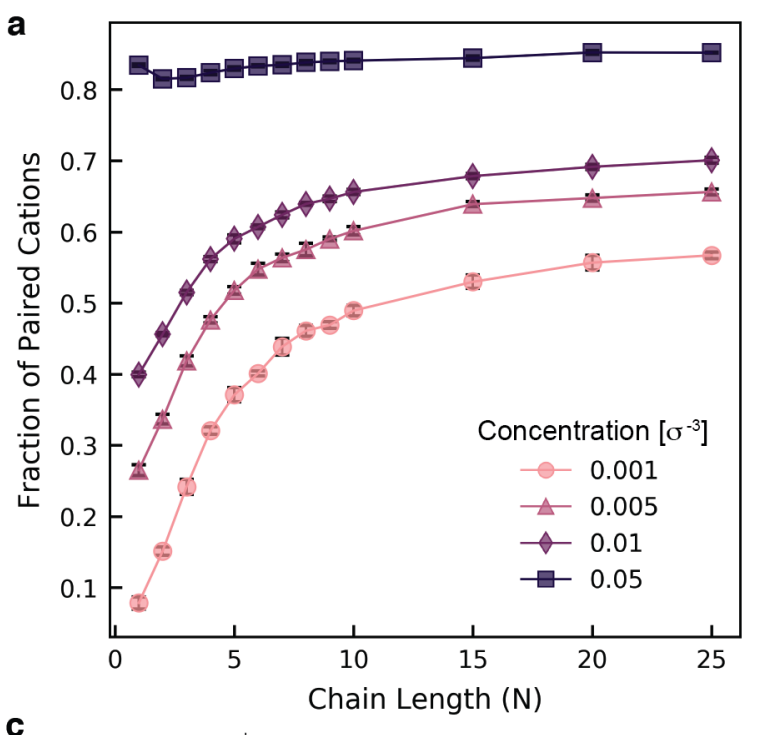

C
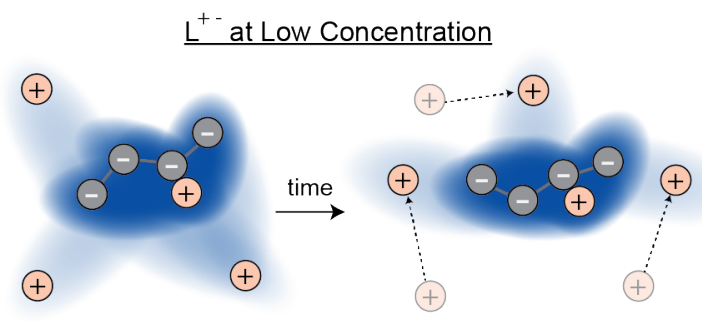

Few, long-lived ion pairs
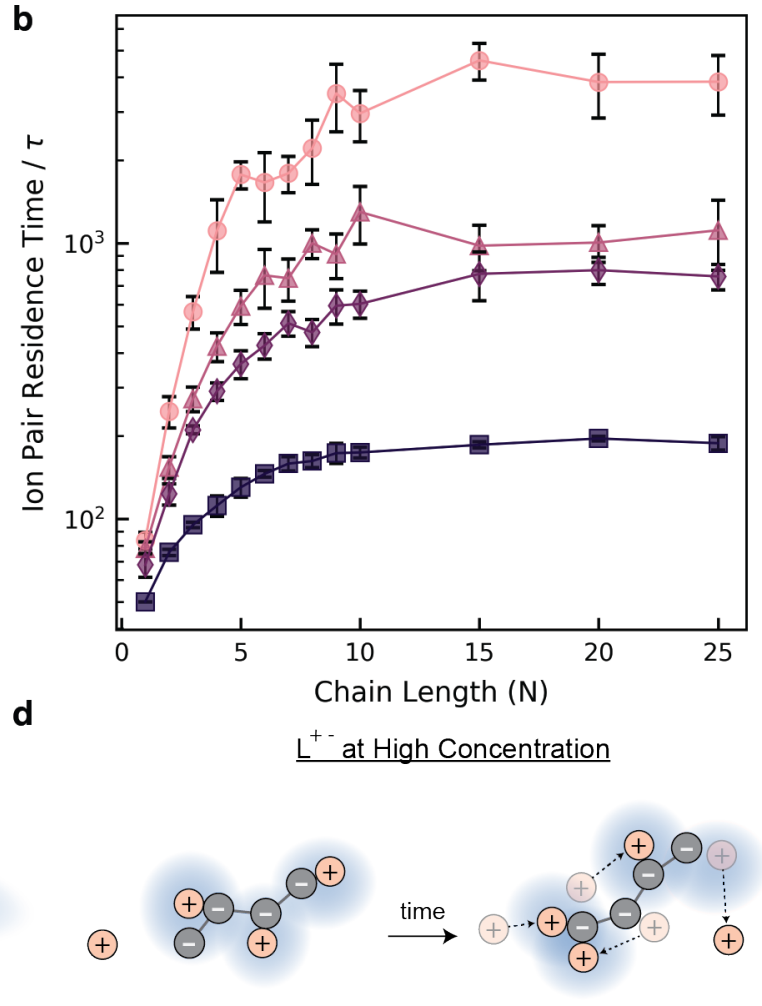

Many, short-lived ion pairs

Figure 4: Ion pairing analysis. (a) Fraction of cations in ion pairs or aggregates based on static structural analysis. (b) Ion pair residence times. (c) and (d) Schematic illustration of cation-anion correlations $\left(L^{+-}\right)$at low $(\mathrm{c})$ and high $(\mathrm{d})$ concentrations. Ion correlations are depicted according to the colormap in Figure 1.

\section{Solvent-free systems}

Given the trend of increasing cation transference number with concentration, the question naturally arises as to whether further increasing concentration may yield polyelectrolytes with $t_{+}$greater than that of the monomeric solution. We can answer this question by considering the limit of a solvent-free system, i.e., a polymerized ionic liquid.

We must first revisit our theoretical framework for the case of a solvent-free system. As mentioned above, a system with $n$ components will have $n(n-1) / 2$ independent transport coefficients. A two-component electrolyte will thus only have one independent transport coefficient. We remind the reader that $\sum_{i} M_{i} L^{i j}=0$ as a consequence of our center-of-mass reference frame, where $M_{i}$ is the molar mass of species $i$. Noting that the masses of all 

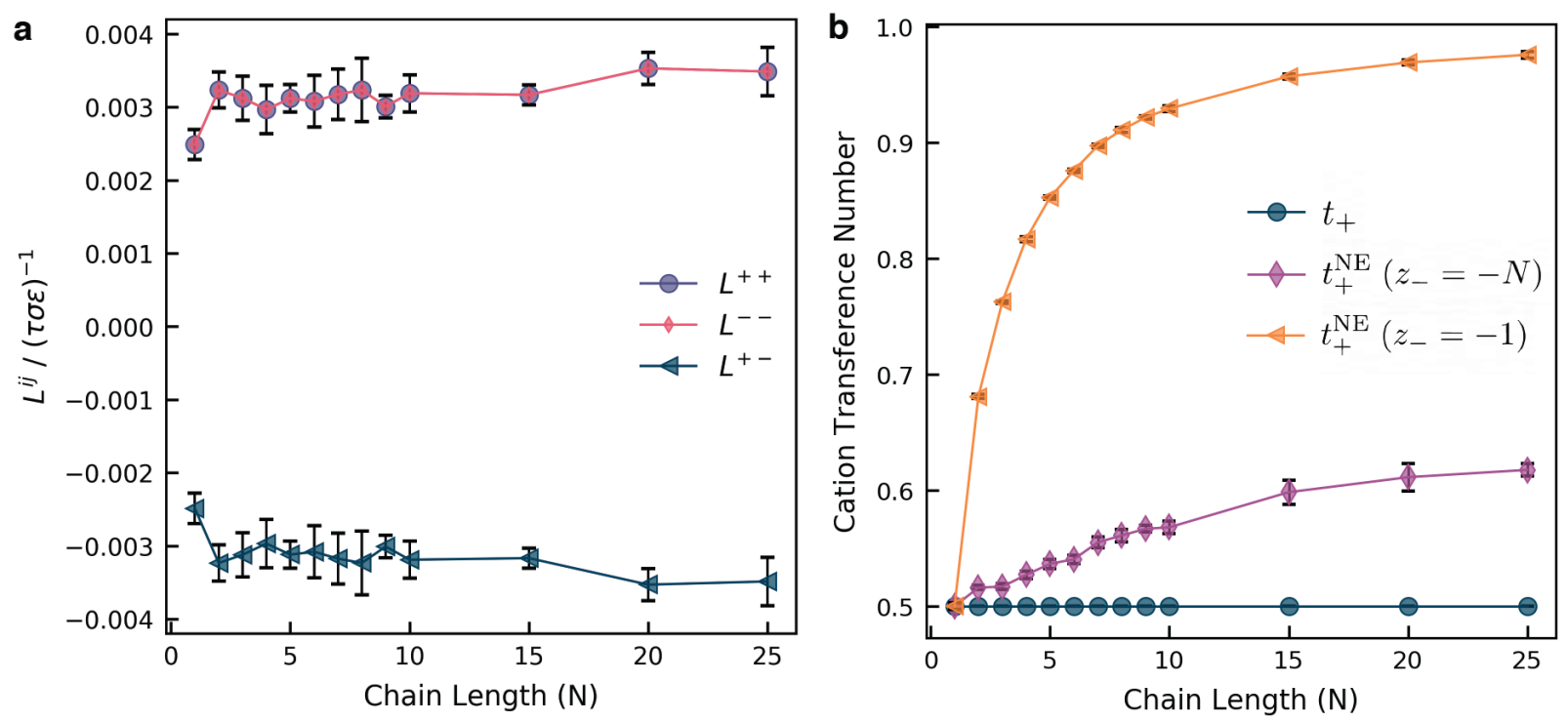

Figure 5: Transport data for the solvent-free solution. (a) Transport coefficients as a function of chain length. The markers for $L^{++}$and $L^{--}$directly overlap. (b) Transference number as a function of chain length, computed rigorously $\left(t_{+}\right)$and using the Nernst-Einstein approximation $\left(t_{+}^{\mathrm{NE}}\right)$.

species in our system are equivalent, we can conclude that $L^{++}=L^{--}=-L^{+-}$in these solvent-free systems. Indeed, the computed transport coefficients follow exactly this relation (Figure 5a; self and distinct transport coefficients for these systems are provided in Figure S9). The implications of this data are twofold. First, $L^{+-}$is necessarily negative in a twocomponent electrolyte, suggesting anti-correlated cation-anion motion. This phenomena has been previously noted in the ionic liquids literature. ${ }^{18,37,38}$ Second, from Eq. (11) it can be shown that the transference number of these solvent-free solutions is determined solely by the ion charges and masses: $t_{+}=z_{+} M_{-} /\left(z_{+} M_{-}-z_{-} M_{+}\right)$. This phenomenon, too, has been noted previously for polymerized ionic liquids ${ }^{18}$ and molten salts. ${ }^{39,40}$ In the polyelectrolyte solutions modeled here where the cation and monomer masses are the same, $t_{+}=0.5$ for all chain lengths. As with the polyelectrolyte solutions with solvent, we observe that the NernstEinstein approximation of the transference number $\left(t_{+}^{\mathrm{NE}}\right)$ drastically overestimates the true $t_{+}$(Figure 5b). It is important to note that the main utility of the transference number is in dictating the extent of concentration gradients in an electrolyte, which limit a battery's rate 
capability. ${ }^{3}$ As electroneutrality dictates that no concentration gradients may be formed in a solvent-free electrolyte, however, the transference number of these systems is of little physical relevance in predicting battery performance. Regardless, even in this superconcentrated limit, we do not observe any polyelectrolyte solutions with cation transference number greater than the conventional monomeric systems.

\section{Conclusions}

In this work, we have computed the Onsager transport coefficients in polyelectrolytes solutions as a function of chain length and concentration using coarse-grained molecular dynamics simulations with explicit solvent. These transport coefficients provide insight into the ion correlations dictating ion motion and allow us to rigorously compute the transference number of the solutions. We demonstrate that the intrinsic anion-anion correlations within chains result in decreasing $t_{+}$as chain length increases and cause substantial deviation between the true $t_{+}$and the ideal solution quantity $t_{+}^{\mathrm{NE}}$. Furthermore, the decrease in $t_{+}$with decreasing concentration can be attributed to stronger cation-anion correlations for more dilute solutions. These stronger correlations are primarily attributed to the longer lifetime of existing ion pairs rather than an increased quantity of ion pairs (defined based on a structural picture of the solution). Even in the case of a solvent-free system (polymerized ionic liquid), we do not observe any polyelectrolyte solutions with transference number greater than that of a conventional monomeric electrolyte. These results suggest that unentangled, short-chain polyelectrolyte solutions may not be useful as high $t_{+}$alternatives to conventional LIB electrolytes. It is possible that more promising systems may be developed using entangled or cross-linked polymer chains, where polymer transport will slow down more dramatically, as well as systems where only a fraction of the monomers are negatively charged. Balancing the increased viscosity and decreased total conductivity inherent to these approaches will be a challenging subject of future work. 


\section{Methods}

\section{Coarse-grained polyelectrolyte model}

Polyanions were represented by the Kremer-Grest bead-spring chain model. ${ }^{24,25}$ Adjacent beads, each representing one monomer, interacted via finitely extensible nonlinear elastic (FENE) bonds, where interaction energies take the form

$$
U_{\mathrm{FENE}}(r)=-0.5 K R_{0}^{2} \ln \left[1-\left(\frac{r}{R_{0}}\right)^{2}\right]
$$

Here $r$ is the distance between two monomers, the spring constant $K=30 \varepsilon / \sigma^{2}$, and the maximum extension of the bond $R_{0}=1.5 \sigma$. These bonding parameters yield an average bond length of $0.97 \sigma$ and avoid unphysical crossing of bonds. ${ }^{41,42}$

Counterions and solvent molecules were modeled explicitly as charged and neutral beads, respectively. Although it increases the computational cost of the simulations, including explicit solvent has been shown in several works to be crucial for accurately capturing dynamics in the solution. ${ }^{43-45}$ All particles (monomers, cations, and solvent) were subject to a truncated-shifted Lennard-Jones (LJ) potential to account for excluded volume,

$$
U_{\mathrm{LJ}}(r)=\left\{\begin{array}{ll}
4 \varepsilon_{\mathrm{LJ}}\left[\left(\frac{\sigma_{\mathrm{LJ}}}{r}\right)^{12}-\left(\frac{\sigma_{\mathrm{LJ}}}{r}\right)^{6}-\left(\frac{\sigma_{\mathrm{LJ}}}{r_{\mathrm{cut}}}\right)^{12}+\left(\frac{\sigma_{\mathrm{LJ}}}{r_{\mathrm{cut}}}\right)^{6}\right] & r \leq r_{\text {cut }} \\
0 & r>r_{\text {cut }}
\end{array} .\right.
$$

For all interactions, $\varepsilon_{\mathrm{LJ}}=\varepsilon$ (the LJ unit of energy) and $\sigma_{\mathrm{LJ}}=\sigma$, where $\sigma$ is the LJ unit of distance and the diameter of each of the beads in the system. We choose $r_{\text {cut }}=2^{1 / 6} \sigma$, which yields good solvent conditions. ${ }^{46}$ All species are given unit mass.

Cations and (poly)anions were additionally subject to the Coulomb potential,

$$
U_{\text {Coulomb }}(r)=\frac{z_{i} z_{j} e^{2}}{4 \pi \epsilon_{0} \epsilon_{r} r}=k_{\mathrm{B}} T \frac{l_{\mathrm{B}} z_{i} z_{j}}{r}
$$


where $e$ is the elementary charge, $\epsilon_{0}$ is the vacuum permittivity, $\epsilon_{r}$ is the dielectric constant of the medium, and $z_{i}$ is the charge of species $i$. Each cation was assigned $z_{+}=+1$ and each monomer was given $z_{-}=-1$. The quantity $l_{\mathrm{B}}=e^{2} /\left(4 \pi \epsilon_{0} \epsilon_{r} k_{\mathrm{B}} T\right)$ is the Bjerrum length, which defines the length scale at which the energy of electrostatic interactions is equal to the thermal energy, $k_{\mathrm{B}} T$. As the simulations in this work capture a wide range of polymer concentrations (from the semi-dilute regime to the limit of a solvent-free system), the dielectric constant was varied linearly as a function of fraction of solvent. ${ }^{47}$ The lower limit of the dielectric constant, corresponding to a system with only solvent, was chosen to mimic the dielectric properties of water with a Bjerrum length of $7.1 \AA$. We follow the common procedure ${ }^{44,46}$ of mapping the average distance between polymers beads, $0.97 \sigma$, to the size of a polystyrene sulfonate monomer, $2.5 \AA$, which yields a Bjerrum length of $l_{\mathrm{B}}=7.1 \AA \times(0.97 \sigma / 2.5 \AA)=2.75 \sigma$. The upper limit of the Bjerrum length for the system with no solvent was chosen to be $30 \sigma$, in the range commonly chosen to study melts of charged polymers. ${ }^{27,41}$

Note that with the length scale mapping of $2.5 \AA \approx 0.97 \sigma$, the ion concentrations simulated in this work $\left(0.001,0.005,0.01\right.$, and $\left.0.05 \sigma^{-3}\right)$ can be roughly mapped to concentrations of $0.1,0.48,0.97$, and $4.8 \mathrm{M}$.

\section{Simulation details}

Polymer chains were initially prepared using a self-avoiding random walk. The polymers, cations, and solvent particles were randomly packed into a cubic simulation box using PACK$\mathrm{MOL}^{48}$ at a density of $0.8 \sigma^{-3}$, a value in the range commonly used to study both dilute polyelectrolyte solutions as well as polymer melts. ${ }^{27,41,49-52}$ With the exception of the systems at the lowest concentration studied, $0.001 \sigma^{-3}$, each simulation consisted of approximately 40,000 particles, with the exact number varied slightly to precisely reach the target concentration. It was verified that doubling the number of particles in the simulation box did not have any appreciable effect on the computed transport properties. At $0.001 \sigma^{-3}$, however, 
finite size effects were more pronounced due to the smaller number of polymer chains in the system and the larger charge screening length. Simulations at this concentration were run with 80,000 particles, with one run using 120,000 particles verifying that there were no appreciable finite size effects with this larger box size. The systems with the longest chain length $(N=25)$ had $4,10,20$, and 100 polymer chains for concentrations of $0.001,0.005$, 0.01 , and $0.05 \sigma^{-3}$, respectively.

Molecular dynamics (MD) simulations were performed using the LAMMPS code. ${ }^{53,54}$ The as-prepared system was equilibrated using a conjugate gradient energy minimization. Simulations were run for a total of $10^{7}$ steps, with a time step of $0.005 \tau$. The first $4 \times 10^{5}$ steps $(2000 \tau)$ were considered to be an equilibration period and not used for data analysis. This equilibration period was long enough for the polymer end-to-end vector autocorrelation function to decay to less than 0.1 for all systems (Figure S10), suggesting that there were minimal effects of the initial system configuration by the time data collection began. We further confirmed that the polymers' radii of gyration and the total system energy had stabilized by the end of the equilibration period.

The equations of motion were numerically integrated using the velocity-Verlet algorithm, with periodic boundary conditions in all three spatial directions. A Nosé-Hoover style thermostat was used to maintain the temperature of the system at $k_{\mathrm{B}} T / \varepsilon=1$. Long-range Coulombic interactions were evaluated using the PPPM method. ${ }^{55}$

\section{Data analysis}

Transport coefficients were computed using Eq. (4):

$$
L^{i j}=\frac{1}{6 k_{\mathrm{B}} T V} \lim _{t \rightarrow \infty} \frac{d}{d t}\left\langle\sum_{\alpha}\left[\boldsymbol{r}_{i}^{\alpha}(t)-\boldsymbol{r}_{i}^{\alpha}(0)\right] \cdot \sum_{\beta}\left[\boldsymbol{r}_{j}^{\beta}(t)-\boldsymbol{r}_{j}^{\beta}(0)\right]\right\rangle
$$

The angular brackets denote averaging over all time origins within the trajectory. The self terms $L_{\text {self }}^{i i}$ (and thus the diffusion coefficients, by Eq. (8)) are computed in an analogous 
manner using Eq. (6), and the distinct terms were computed by $L_{\text {distinct }}^{i i}=L^{i i}-L_{\text {self }}^{i i}$ All experimentally-relevant quantities such as the transference number were computed from $L^{i j}$ using Eqs. (9)-(11).

In order to capture true diffusive transport, the term in angular brackets of Eqs. (4) or (6) must be linear with respect to time, i.e. $\left\langle\sum_{\alpha}\left[\boldsymbol{r}_{i}^{\alpha}(t)-\boldsymbol{r}_{i}^{\alpha}(0)\right] \cdot \sum_{\beta}\left[\boldsymbol{r}_{j}^{\beta}(t)-\boldsymbol{r}_{j}^{\beta}(0)\right]\right\rangle \propto t^{\beta}$ and $\sum_{\alpha}\left\langle\left[\boldsymbol{r}_{i}^{\alpha}(t)-\boldsymbol{r}_{i}^{\alpha}(0)\right]^{2}\right\rangle \propto t^{\beta}$, with $\beta=1$. For most simulations in this work, $\beta$ was computed to be between 0.94 and 1.05, with analysis performed over at least a decade of time. This suggests that the simulations have been run long enough to reach and adequately sample the diffusive regime. Representative data demonstrating this linear behavior is given in Fig. S4. There are a two classes of exceptions in which $\beta$ deviates more substantially from unity. The first is for $L^{+-}$in the monomer simulations $(N=1)$ at the three lowest concentrations studied, in which case $\beta$ became as low as 0.84 . This deviation from linearity is due to the small overall magnitude of $L^{+-}$, which results in more noise in the fitting procedure. As $L^{+-} \approx 0$ for these systems, the fact that we do not observe linear behavior does not impact any of our conclusions. The second case where $\beta$ departs from unity is for $L_{\text {self }}^{--}$in the solvent-free systems for chain lengths of 15,20 , and 25 , where $\beta$ reached as low as 0.73 . This sub-diffusive behavior can be attributed to anion motion corresponding to intra-chain degrees of freedom, such as chain rotations. While the values of $L_{\text {self }}^{--}$for these three chain lengths presented in Fig. S9 can not be rigorously interpreted as transport coefficients due to this deviation from linearity, this does not affect any of the conclusions drawn in this work.

The extent of ion pairing as shown in Figure 4a was evaluated using a distance criterion: all cations within a given cutoff distance of any anion were considered paired. This cutoff distance was chosen as the first minimum of the cation-anion radial distribution function (1.6 $\sigma$, see Figure S12), which was consistent across all chain lengths and concentrations. The residence times of ion pairs given in Figure 4b were evaluated by computing the lifetime correlation function $P_{\alpha \beta}(t)=\left\langle H_{\alpha \beta}(t) H_{\alpha \beta}(0)\right\rangle$, where $H_{\alpha \beta}(t)$ is one if particles $\alpha$ and $\beta$ are 
neighbors at time $t$ and zero otherwise. ${ }^{16,29-31}$ Two particles are deemed neighbors if they fall within a distance cutoff, chosen based on the radial distribution function to be $2.5 \sigma$ to coincide with the minimum after the second solvation shell of the ions. This distance cutoff was larger than that used for the static evaluation of ion pairing, as we observed that ions moved very frequently back and forth between the first and second solvation shells. The residence time was defined as the time for $P_{\alpha \beta}(t)$ to decay to $10 \%$ of its original value.

Error bars throughout this text are given as the standard deviation of five independent replicates. Trajectories were analyzed using an in-house code which utilized the Python package MDAnalysis. ${ }^{56,57}$ Code is available at https://github.com/kdfong/transport-coefficientsMSD.

No unexpected or unusually high safety hazards were encountered.

\section{Acknowledgement}

K.D.F. acknowledges support from NSF GRFP under Grant no. DGE 1752814. This work was supported by the Assistant Secretary for Energy Efficiency and Renewable Energy, Vehicle Technologies Office, of the U.S. Department of Energy under Contract DE-AC0205CH11231, under the Advanced Battery Materials Research (BMR) Program. This research used resources of the Savio computational cluster resource provided by the Berkeley Research Computing program at the University of California, Berkeley (supported by the UC Berkeley Chancellor, Vice Chancellor for Research, and Chief Information Officer).

\section{Supporting Information Available}

Additional transport coefficient data and discussion; conductivity, mobility, and diffusion coefficients; characterization of polymer size and relaxation time; radial distribution functions. 


\section{References}

(1) Doyle, M.; Fuller, T. F.; Newman, J. The importance of the lithium ion transference number in lithium/polymer cells. Electrochimica Acta 1994, 39, 2073-2081.

(2) Newman, J.; Thomas-Alyea, K. E. Electrochemical Systems, 3rd ed.; John Wiley \& Sons, Inc., 2004.

(3) Diederichsen, K. M.; McShane, E. J.; McCloskey, B. D. Promising routes to a high $\mathrm{Li}^{+}$ transference number electrolyte for lithium ion batteries. ACS Energy Letters 2017, 2, $2563-2575$.

(4) Zhang, H.; Li, C.; Piszcz, M.; Coya, E.; Rojo, T.; Rodriguez-Martinez, L. M.; Armand, M.; Zhou, Z. Single lithium-ion conducting solid polymer electrolytes: Advances and perspectives. Chemical Society Reviews 2017, 46, 797-815.

(5) Jeong, K.; Park, S.; Lee, S.-Y. Revisiting polymeric single lithium-ion conductors as an organic route for all-solid-state lithium ion and metal batteries. Journal of Materials Chemistry A 2019, 7, 1917-1935.

(6) Bocharova, V.; Sokolov, A. Perspectives for polymer electrolytes: A view from fundamentals of ionic conductivity. Macromolecules 2020, 53, 4141-4157.

(7) Strauss, E.; Menkin, S.; Golodnitsky, D. On the way to high-conductivity single lithiumion conductors. Journal of Solid State Electrochemistry 2017, 21, 1879-1905.

(8) Kreuer, K. D.; Wohlfarth, A.; De Araujo, C. C.; Fuchs, A.; Maier, J. Single alkaline-ion $\left(\mathrm{Li}^{+}, \mathrm{Na}^{+}\right)$conductors by ion exchange of proton-conducting ionomers and polyelectrolytes. ChemPhysChem 2011, 12, 2558-2560.

(9) Smiatek, J.; Wohlfarth, A.; Holm, C. The solvation and ion condensation properties for sulfonated polyelectrolytes in different solvents - A computational study. New Journal of Physics 2014, 16, 025001. 
(10) Buss, H. G.; Chan, S. Y.; Lynd, N. A.; McCloskey, B. D. Nonaqueous polyelectrolyte solutions as liquid electrolytes with high lithium ion transference number and conductivity. ACS Energy Letters 2017, 2, 481-487.

(11) Diederichsen, K. M.; Fong, K. D.; Terrell, R. C.; Persson, K. A.; McCloskey, B. D. Investigation of solvent type and salt addition in high transference number nonaqueous polyelectrolyte solutions for lithium ion batteries. Macromolecules 2018, 51, 8761-8771.

(12) Diederichsen, K. M.; Terrell, R. C.; McCloskey, B. D. Counterion transport and transference number in aqueous and nonaqueous short-chain polyelectrolyte solutions. The Journal of Physical Chemistry B 2019, 123, 10858-10867.

(13) Diederichsen, K. M.; McCloskey, B. D. Electrolyte additives to enable nonaqueous polyelectrolyte solutions for lithium ion batteries. Molecular Systems Design 8 Engineering 2020, 5, 91-96.

(14) Dewing, B. L.; Bible, N. G.; Ellison, C. J.; Mahanthappa, M. K. Electrochemically stable, high transference number lithium bis (malonato) borate polymer solution electrolytes. Chemistry of Materials 2020, 32, 3794-3804.

(15) Valøen, L. O.; Reimers, J. N. Transport properties of LiPF ${ }_{6}$-based Li-ion battery electrolytes. Journal of The Electrochemical Society 2005, 152, A882.

(16) Fong, K. D.; Self, J.; Diederichsen, K. M.; Wood, B. M.; McCloskey, B. D.; Persson, K. A. Ion transport and the true transference number in nonaqueous polyelectrolyte solutions for lithium ion batteries. ACS Central Science 2019, 5, 1250-1260.

(17) Qian, W.; Texter, J.; Yan, F. Frontiers in poly(ionic liquid)s: Syntheses and applications. Chemical Society Reviews 2017, 46, 1124-1159.

(18) Zhang, Z.; Wheatle, B. K.; Krajniak, J.; Keith, J. R.; Ganesan, V. Ion mobilities, 
transference numbers, and inverse Haven ratios of polymeric ionic liquids. ACS Macro Letters 2019, 9, 84-89.

(19) Yu, Y.; Lu, F.; Sun, N.; Wu, A.; Pan, W.; Zheng, L. Single lithium-ion polymer electrolytes based on poly(ionic liquid)s for lithium-ion batteries. Soft Matter 2018, 14, 6313-6319.

(20) Muthukumar, M. 50th Anniversary perspective: A perspective on polyelectrolyte solutions. Macromolecules 2017, 50, 9528-9560.

(21) Dobrynin, A. V. Polymer Science: A Comprehensive Reference, 10 Volume Set; Elsevier, 2012; Vol. 1; pp 81-132.

(22) Fong, K. D.; Bergstrom, H. K.; McCloskey, B. D.; Mandadapu, K. K. Transport phenomena in electrolyte solutions: Non-equilibrium thermodynamics and statistical mechanics. arXiv preprint arXiv:2006.16164 2020,

(23) Bird, R. B.; Klingenberg, D. J. Multicomponent diffusion — A brief review. Advances in Water Resources 2013, 62, 238-242.

(24) Kremer, K.; Grest, G. S. Dynamics of entangled linear polymer melts: A moleculardynamics simulation. The Journal of Chemical Physics 1990, 92, 5057-5086.

(25) Grest, G. S.; Kremer, K. Molecular dynamics simulation for polymers in the presence of a heat bath. Physical Review A 1986, 33, 3628.

(26) Hoy, R. S.; Foteinopoulou, K.; Kröger, M. Topological analysis of polymeric melts: Chain-length effects and fast-converging estimators for entanglement length. Physical Review E 2009, 80, 031803.

(27) Hall, L. M.; Seitz, M. E.; Winey, K. I.; Opper, K. L.; Wagener, K. B.; Stevens, M. J.; Frischknecht, A. L. Ionic aggregate structure in ionomer melts: Effect of molecular 
architecture on aggregates and the ionomer peak. Journal of the American Chemical Society 2012, 134, 574-587.

(28) Nyquist, R. M.; Ha, B. Y.; Liu, A. J. Counterion condensation in solutions of rigid polyelectrolytes. Macromolecules 1999, 32, 3481-3487.

(29) Self, J.; Fong, K. D.; Persson, K. A. Transport in superconcentrated LiPF 6 and $\mathrm{LiBF}_{4} /$ propylene carbonate electrolytes. ACS Energy Letters 2019, 4, 2843-2849.

(30) A joint theoretical/experimental study of the structure, dynamics, and $\mathrm{Li}^{+}$transport in bis([tri]fluoro[methane]sulfonyl)imide [T]FSI-based ionic liquids. Journal of Chemical Physics 2013, 139, 034502.

(31) Borodin, O.; Smith, G. D.; Henderson, W. Li ${ }^{+}$cation environment, transport, and mechanical properties of the LiTFSI doped N-methyl-N-alkylpyrrolidinium ${ }^{+} \mathrm{TFSI}^{-}$ionic liquids. Journal of Physical Chemistry B 2006, 110, 16879-16886.

(32) Joshi, Y.; Kwak, J. C. Transference numbers, polyion mobilities, and charge fractions in aqueous solutions of lithium, sodium, and potassium dextransulfate. Biophysical chemistry 1980, 12, 323-328.

(33) Jordan, D.; Kurucsev, T.; Martin, M. Comparative physical chemical study of isotactic and atactic poly (styrene sulphonic acid) solutions. Part 2. Electrical conductance and transference measurements in salt-free aqueous solutions. Transactions of the Faraday Society 1969, 65, 606-611.

(34) De, R.; Lee, H.; Das, B. Exploring the interactions in binary mixtures of polyelectrolytes: Influence of mixture composition, concentration, and temperature on counterion condensation. Journal of Molecular Liquids 2018, 251, 94-99.

(35) Kuznetsov, I.; Vorontsova, O.; Kozlov, A. Polyelectrolyte properties of biopolymers: 
Conductivity and secondary structure of polyriboadenylic acid and its salts in solutions. Biopolymers: Original Research on Biomolecules 1991, 31, 65-76.

(36) Overbeek, J. T. G. Macromolecular Chemistry-11; Elsevier, 1977; pp 91-101.

(37) Kashyap, H. K.; Annapureddy, H. V.; Raineri, F. O.; Margulis, C. J. How is charge transport different in ionic liquids and electrolyte solutions? The Journal of Physical Chemistry B 2011, 115, 13212-13221.

(38) McDaniel, J. G.; Son, C. Y. Ion correlation and collective dynamics in BMIM/BF $4^{-}$ based organic electrolytes: From dilute solutions to the ionic liquid limit. The Journal of Physical Chemistry B 2018, 122, 7154-7169.

(39) Matsunaga, S.; Koishi, T.; Tamaki, S. Velocity correlation functions and partial conductivities of molten AgI-AgBr by molecular dynamics simulation. Materials Science and Engineering: $A$ 2007, 449, 693-698.

(40) Koishi, T.; Tamaki, S. Partial conductivities of a molten salt based on Langevin equation. Journal of the Physical Society of Japan 1999, 68, 964-971.

(41) Ma, B.; Nguyen, T. D.; Olvera de la Cruz, M. Control of ionic mobility via charge size asymmetry in random ionomers. Nano letters 2019, 20, 43-49.

(42) Chang, R.; Yethiraj, A. Strongly charged flexible polyelectrolytes in poor solvents: Molecular dynamics simulations with explicit solvent. The Journal of chemical physics 2003, 118, 6634-6647.

(43) Yao, N.; Li, J.; Gu, F.; Wang, H.; Liao, Q. Relaxation time spectrum and dynamics of stretched polymer chain in dilute $\theta$ solution: Implicit solvent model versus explicit solvent model. Macromolecular Theory and Simulations 2020, 29, 1900064.

(44) Grass, K.; Holm, C. Polyelectrolytes in electric fields: Measuring the dynamical effective charge and effective friction. Soft Matter 2009, 5, 2079-2092. 
(45) Chang, R.; Yethiraj, A. Dilute solutions of strongly charged flexible polyelectrolytes in poor solvents: Molecular dynamics simulations with explicit solvent. Macromolecules 2006, 39, 821-828.

(46) Micka, U.; Holm, C.; Kremer, K. Strongly charged, flexible polyelectrolytes in poor solvents: Molecular dynamics simulations. Langmuir 1999, 15, 4033-4044.

(47) Hall, D. S.; Self, J.; Dahn, J. Dielectric constants for quantum chemistry and Li-ion batteries: Solvent blends of ethylene carbonate and ethyl methyl carbonate. The Journal of Physical Chemistry C 2015, 119, 22322-22330.

(48) Martínez, L.; Andrade, R.; Birgin, E. G.; Martínez, J. M. PACKMOL: A package for building initial configurations for molecular dynamics simulations. Journal of Computational Chemistry 2009, 30, 2157-2164.

(49) Lo, T. S.; Khusid, B.; Koplik, J. Dynamical clustering of counterions on flexible polyelectrolytes. Physical review letters 2008, 100, 128301.

(50) Chremos, A.; Douglas, J. F. Counter-ion distribution around flexible polyelectrolytes having different molecular architecture. Soft Matter 2016, 12, 2932-2941.

(51) Carrillo, J.-M. Y.; Brown, W. M.; Dobrynin, A. V. Explicit solvent simulations of friction between brush layers of charged and neutral bottle-brush macromolecules. Macromolecules 2012, 45, 8880-8891.

(52) Hall, L. M.; Stevens, M. J.; Frischknecht, A. L. Dynamics of model ionomer melts of various architectures. Macromolecules 2012, 45, 8097-8108.

(53) Plimpton, S. Fast parallel algorithms for short-range molecular dynamics. Journal of Computational Physics 1995, 117, 1-19.

(54) http://lammps.sandia.gov (accessed Aug 11, 2017). 
(55) Toukmaji, A. Y.; Board, J. A. Ewald summation techniques in perspective: A survey. Computer Physics Communications 2003, 95, 73-92.

(56) Michaud-Agrawal, N.; Denning, E. J.; Woolf, T. B.; Beckstein, O. MDAnalysis: A toolkit for the analysis of molecular dynamics simulations. Journal of Computational Chemistry 2011, 32, 2319-2327.

(57) Gowers, R.; Linke, M.; Barnoud, J.; Reddy, T.; Melo, M.; Seyler, S.; Domański, J.; Dotson, D.; Buchoux, S.; Kenney, I.; Beckstein, O. MDAnalysis: A Python package for the rapid analysis of molecular dynamics simulations. Proceedings of the 15th Python in Science Conference. 2016; pp 98-105. 


\title{
Supporting Information:
}

\section{Onsager Transport Coefficients and Transference}

Numbers in Polyelectrolyte Solutions and

Polymerized Ionic Liquids

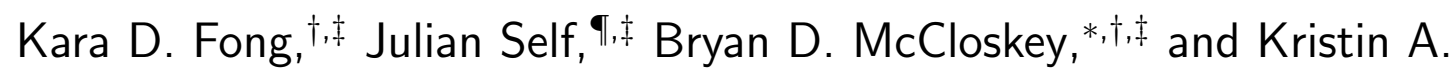 \\ Persson $* \boldsymbol{\Phi}, \ddagger$ \\ $\dagger$ Department of Chemical and Biomolecular Engineering, University of California, Berkeley \\ $\ddagger$ Energy Technologies Area, Lawrence Berkeley National Laboratory \\ IDepartment of Materials Science and Engineering, University of California, Berkeley \\ E-mail: bmcclosk@berkeley.edu; kapersson@lbl.gov
}

\section{Transport coefficients}

\section{Analyzing monomer vs polymer motion}

When computing the anion transport coefficients $\left(L^{--}\right.$and $\left.L^{+-}\right)$in a polyelectrolyte, we may treat either the polymer chain as a whole or individual monomers as the anionic species. The analysis presented in the main text uses the latter convention. In this section, we describe the relationship between the transport coefficients obtained from using each choice and show how the experimentally-relevant quantities (with the exception of the NernstEinstein transference number) are equivalent regardless of the analysis method. We show how combining both choices of anionic species can be used to decouple correlations between 
ions within a given chain and those between ions on different chains (intra- versus inter-chain interactions).

Let us denote the transport coefficients obtained by treating individual monomers as the anion with a subscript " $\mathrm{m}$ ", and those obtained using the whole polymer chain with a subscript "p". The transport coefficients presented in the main text are $L_{\mathrm{m}}^{i j}$. In order to derive relations between $L_{\mathrm{m}}^{i j}$ and $L_{\mathrm{p}}^{i j}$, we begin by writing the Green-Kubo relation for $L_{\mathrm{p}}^{--}$ as

$$
L_{\mathrm{p}}^{--}=\frac{1}{6 k_{\mathrm{B}} T V} \lim _{t \rightarrow \infty} \frac{d}{d t}\left\langle\sum_{a}\left[\boldsymbol{r}_{a}(t)-\boldsymbol{r}_{a}(0)\right] \cdot \sum_{b}\left[\boldsymbol{r}_{b}(t)-\boldsymbol{r}_{b}(0)\right]\right\rangle .
$$

Here we use the indices $a$ and $b$ to denote a sum over each polymer chain, whereas we use the indices $\alpha$ and $\beta$ to denote individual monomers. The quantity $\boldsymbol{r}_{a}$ is the center-of-mass position of the chain relative to the center of mass of the entire system, which can be written as $\boldsymbol{r}_{a}=\frac{1}{N} \sum_{\alpha} \boldsymbol{r}_{a}^{\alpha}$, noting that the masses of all monomers are equal. The quantity $\boldsymbol{r}_{a}^{\alpha}$ is the position (relative to the center of mass of the entire system) of monomer $\alpha$ on chain $a$. Equation (S1) may be written in terms of individual monomer positions as

$$
L_{\mathrm{p}}^{--}=\frac{1}{6 k_{\mathrm{B}} T V N^{2}} \lim _{t \rightarrow \infty} \frac{d}{d t}\left\langle\sum_{a} \sum_{\alpha}\left[\boldsymbol{r}_{a}^{\alpha}(t)-\boldsymbol{r}_{a}^{\alpha}(0)\right] \cdot \sum_{b} \sum_{\beta}\left[\boldsymbol{r}_{b}^{\beta}(t)-\boldsymbol{r}_{b}^{\beta}(0)\right]\right\rangle
$$

Comparing to Eq. (4) in the main text, we can conclude that

$$
L_{\mathrm{p}}^{--}=\frac{1}{N^{2}} L_{\mathrm{m}}^{--}
$$

Similar arguments allow us to relate $L_{\mathrm{p}}^{+-}$and $L_{\mathrm{m}}^{+-}$via

$$
L_{\mathrm{p}}^{+-}=\frac{1}{N} L_{\mathrm{m}}^{+-} .
$$

We can also relate the self anion transport coefficients, $L_{\mathrm{m}, \text { self }}^{--}$and $L_{\mathrm{p}, \text { self }}^{--}$, by assuming that the self-diffusion coefficients of the polymer and monomer will be identical over long enough time scales, i.e., $D_{-, \mathrm{p}}=D_{-, \mathrm{m}}$. Further, note that the concentrations of polymer and 
monomer are related by by $c_{m}=N c_{p}$. Using Eq. (8) of the main text, we can thus conclude that

$$
L_{\mathrm{p}, \mathrm{self}}^{--}=\frac{1}{N} L_{\mathrm{m}, \mathrm{self}}^{--}
$$

As mentioned in the main text, the distinct anion transport coefficients obtained from analyzing monomer motion, $L_{\mathrm{m}, \text { distinct }}^{--}$, captures two forms of anion-anion correlations: those between anions on the same chain (intra-chain) and those between anions on different chains (inter-chain), i.e., $L_{\mathrm{m} \text {,distinct }}^{--}=L_{\mathrm{m} \text {,inter-chain }}^{--}+L_{\mathrm{m} \text {,intra-chain }}^{--}$In contrast, the distinct anion transport coefficient from analyzing polymer center-of-mass motion gives only inter-chain correlations: $L_{\mathrm{p}, \text { distinct }}^{--}=L_{\mathrm{p}, \text { inter-chain }}^{--}$Thus, if we seek to compare the relative magnitude of inter- and intra-chain correlations, we may compute $L_{\mathrm{m} \text {,inter-chain }}^{--}=N^{2} L_{\mathrm{p} \text {,distinct }}^{--}$and $L_{\mathrm{m}, \text { intra-chain }}^{--}=L_{\mathrm{m}, \text { distinct }}^{--}-L_{\mathrm{m} \text {,inter-chain }}^{--}$These data are shown in Figure S5.

Using Eqs. (S3) and (S4), it is easy to demonstrate that the ionic conductivity, cation and anion mobilities, and transference number are equivalent regardless of whether the motion of the monomer $\left(L_{\mathrm{m}}^{i j}\right)$ or polymer center of mass $\left(L_{\mathrm{p}}^{i j}\right)$ is analyzed. Doing so requires us to note that the charge valencies of the polymer and monomer are related by the degree of polymerization $N$, i.e., $z_{\mathrm{p}}=N z_{\mathrm{m}}$. For example, by Eq. (9) the conductivity is

$$
\begin{aligned}
\kappa & =F^{2} \sum_{i} \sum_{j} L^{i j} z_{i} z_{j}=F^{2}\left(L^{++} z_{+}^{2}+L_{\mathrm{p}}^{--} z_{\mathrm{p}}^{2}-2 L_{\mathrm{p}}^{+-} z_{+} z_{\mathrm{p}}\right) \\
& =F^{2}\left(L^{++} z_{+}^{2}+\left(L_{\mathrm{m}}^{--} / N^{2}\right)\left(N z_{\mathrm{m}}\right)^{2}-2\left(L_{\mathrm{m}}^{+-} / N\right) z_{+}\left(N z_{\mathrm{m}}\right)\right) \\
& =F^{2}\left(L^{++} z_{+}^{2}+L_{\mathrm{m}}^{--} z_{\mathrm{m}}^{2}-2 L_{\mathrm{m}}^{+-} z_{+} z_{\mathrm{m}}\right) .
\end{aligned}
$$

The only experimentally-relevant quantity which will change depending on whether we treat the monomer or polymer as the anionic species is $t_{+}^{\mathrm{NE}}=\frac{z_{+} D_{+}}{z_{+} D_{+}-z_{-} D_{-}}$. The diffusion coefficients of the cation and anion will be equivalent regardless of whether the monomer or polymer center of mass is considered, but the anionic charge valence $z_{-}$will change. Using $L_{\mathrm{p}}^{i j}$ corresponds to including $z_{-}=-N$ in the equation, which will yield the $t_{+}^{\mathrm{NE}}$ values shown in Figure 2b, whereas with $L_{\mathrm{m}}^{i j}, z_{-}=-1$ should be used, as in Figure 2c. As analyzing 
polymer center-of-mass motion implicitly captures intra-chain correlations, $t_{+}^{\mathrm{NE}}\left(z_{-}=-N\right)$ will provide a much more reasonable estimate for the true cation transference number than $t_{+}^{\mathrm{NE}}\left(z_{-}=-1\right)$, as is clear from Figure 2.
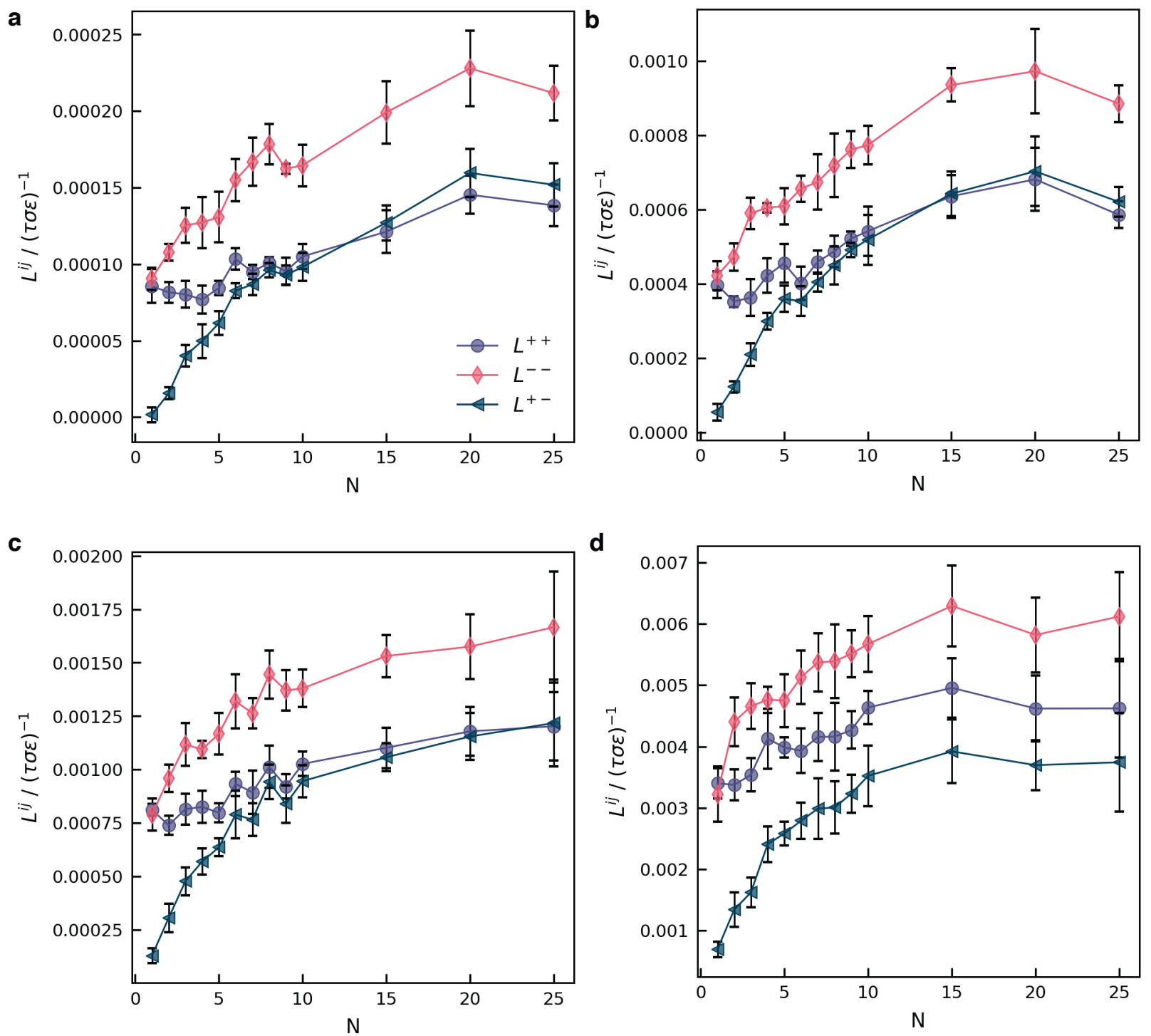

Figure S1: Transport coefficients as a function of chain length for concentrations of (a) 0.001, (b) 0.005 , (c) 0.01 , and (d) $0.05 \sigma^{-3}$. 

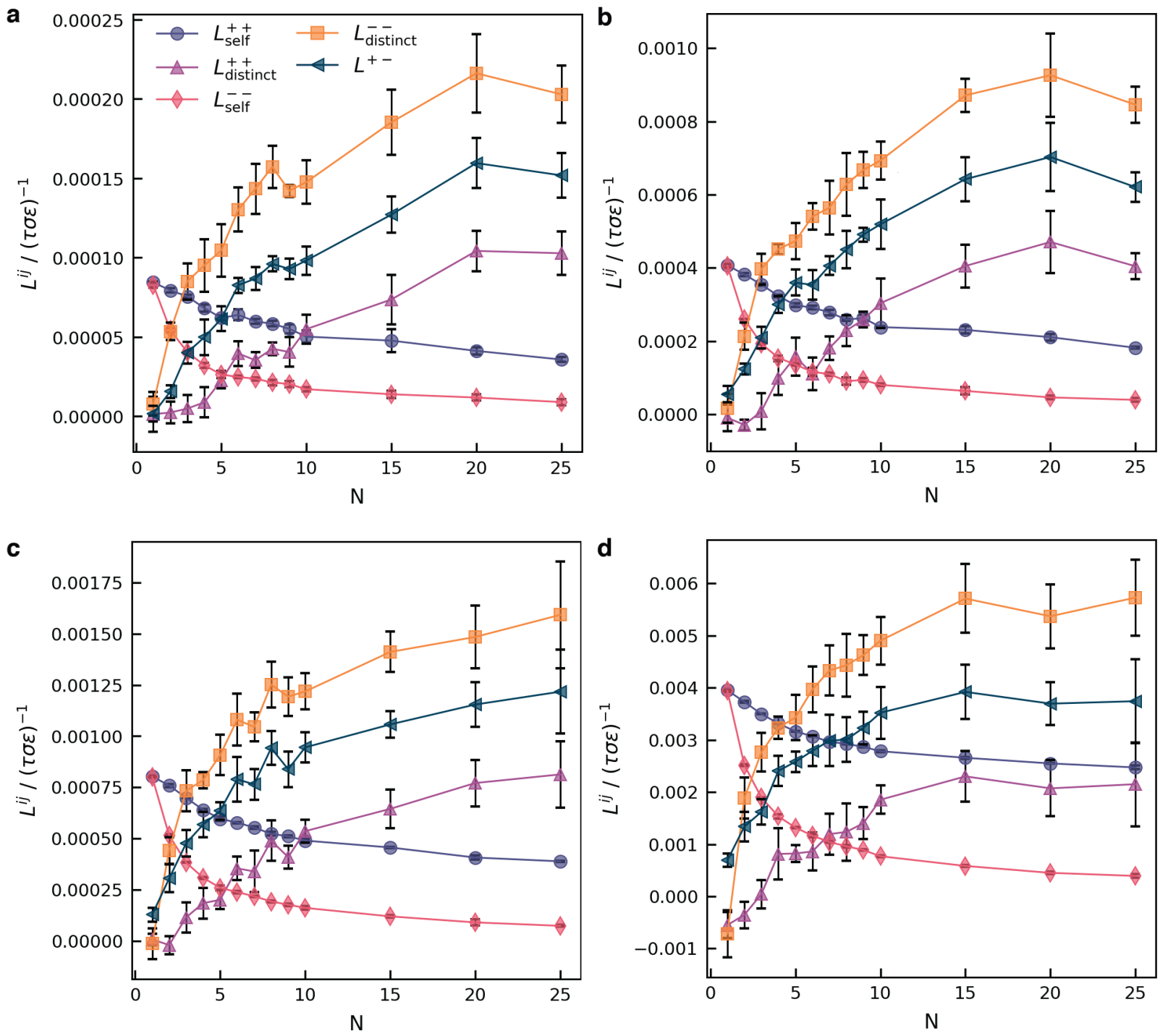

Figure S2: Transport coefficients, with diagonal terms decomposed into self and distinct contributions, as a function of chain length for concentrations of (a) 0.001, (b) 0.005, (c) 0.01 , and (d) $0.05 \sigma^{-3}$. 

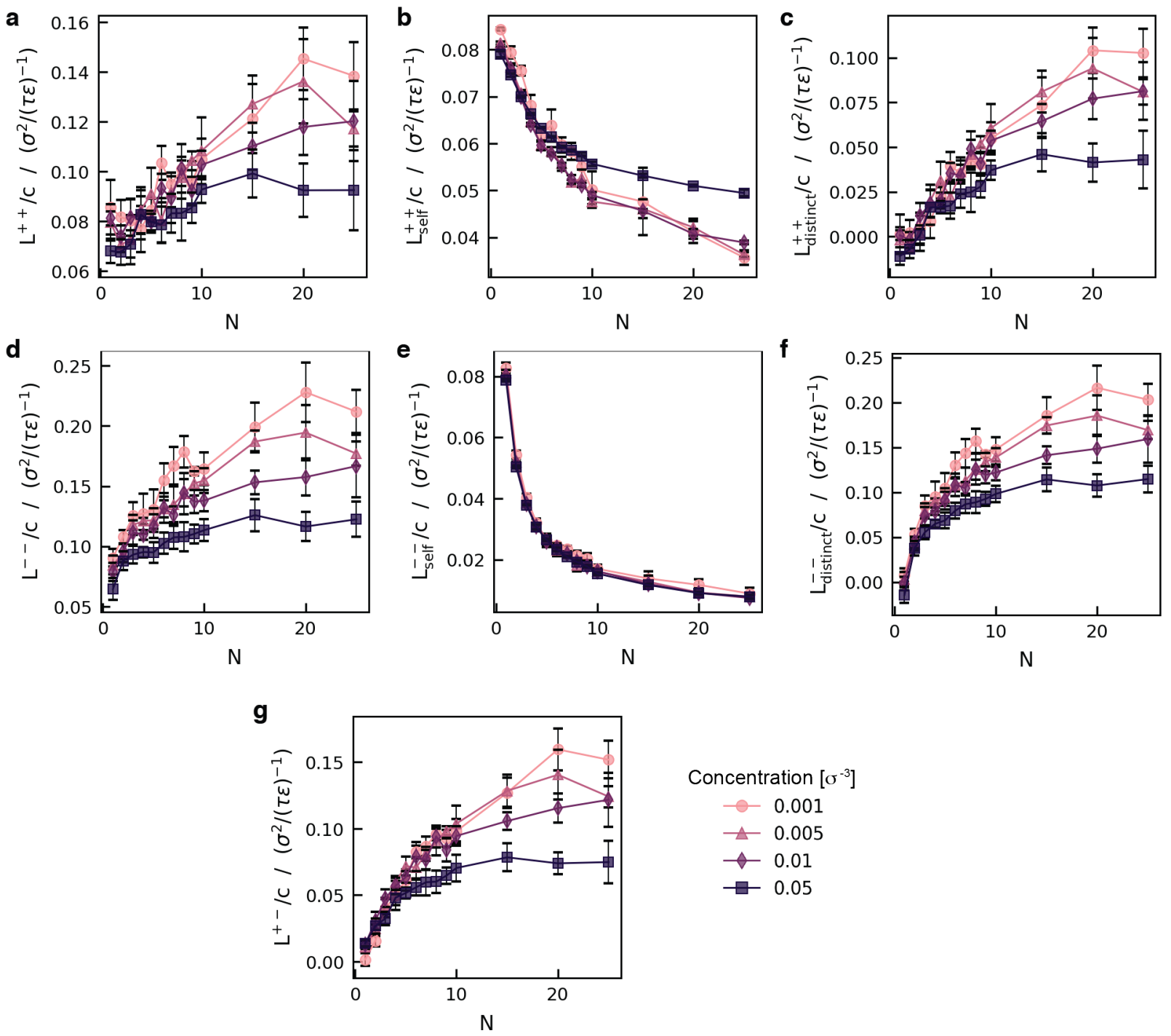

Figure S3: Each of the transport coefficients divided by concentration as a function of chain length. (a) $L^{++} / c$, (b) $L_{\text {self }}^{++} / c$, (c) $L_{\text {distinct }}^{++} / c$, (d) $L^{--} / c$, (e) $L_{\text {self }}^{--} / c$, (f) $L_{\text {distinct }}^{--} / c$, and (g) $L^{+-} / c$. 

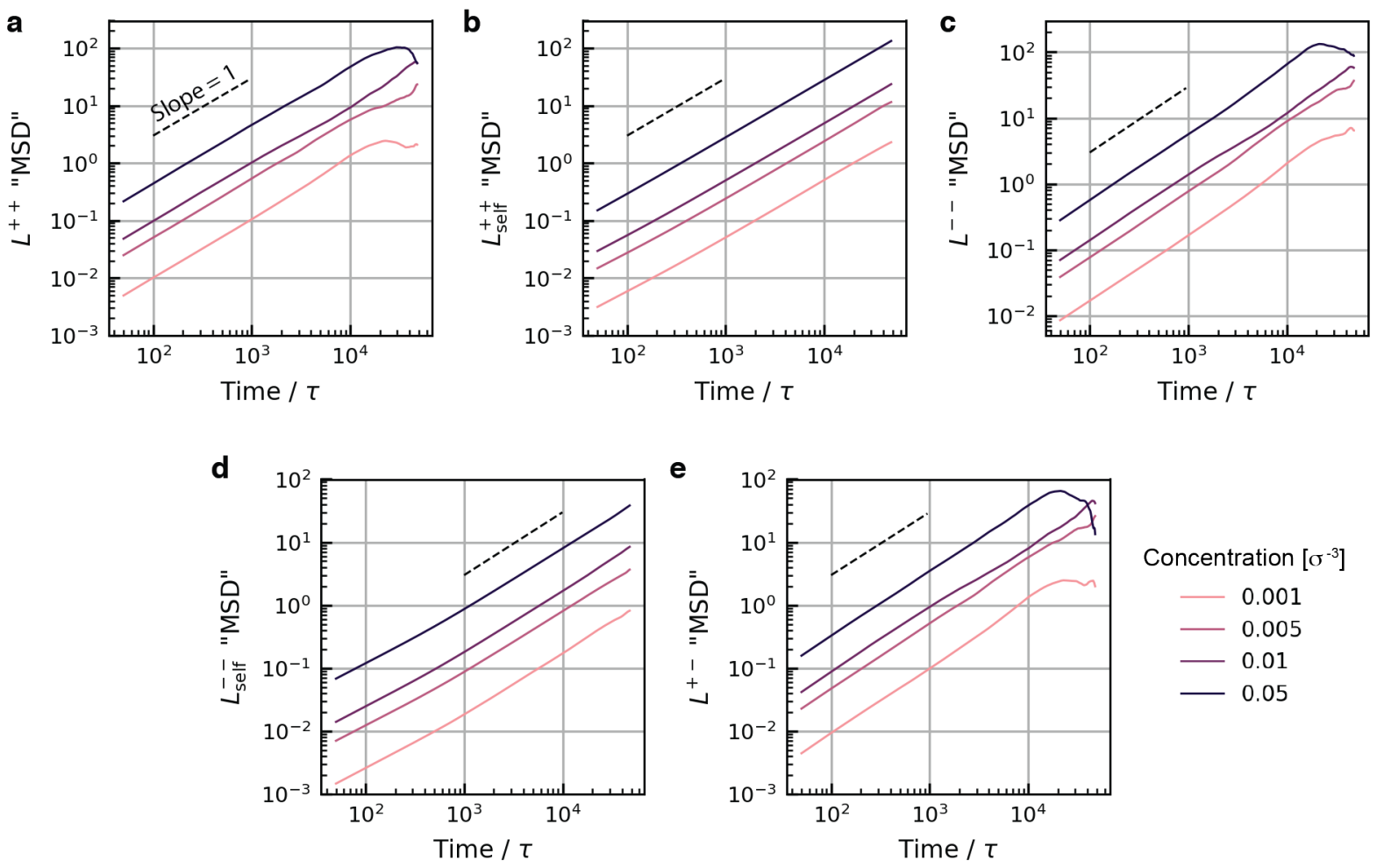

Figure S4: Representative examples of data used to compute $L^{i j}$ and $L_{\text {self }}^{i i}$ using Eqs. (4) and (6), respectively. The quantity plotted is $\left\langle\sum_{\alpha}\left[\boldsymbol{r}_{i}^{\alpha}(t)-\boldsymbol{r}_{i}^{\alpha}(0)\right] \cdot \sum_{\beta}\left[\boldsymbol{r}_{j}^{\beta}(t)-\boldsymbol{r}_{j}^{\beta}(0)\right]\right\rangle$ for computing $L^{i j}$ or $\sum_{\alpha}\left\langle\left[\boldsymbol{r}_{i}^{\alpha}(t)-\boldsymbol{r}_{i}^{\alpha}(0)\right]^{2}\right\rangle$ for $L_{\text {self }}^{i i}$. We refer to these quantities the "MSD", although technically only the latter is actually a mean squared displacement. We show data for (a) $L^{++}$, (b) $L_{\text {self }}^{++}$, (c) $L^{--}$, (d) $L_{\text {self }}^{--}$, and (e) $L^{+-}$. All remaining transport quantities, including the distinct transport coefficients and experimentally-relevant quantities, are computed from these five fits. Data for these plots is for a chain length of $N=10$. Dashed black lines show a slope of one. 


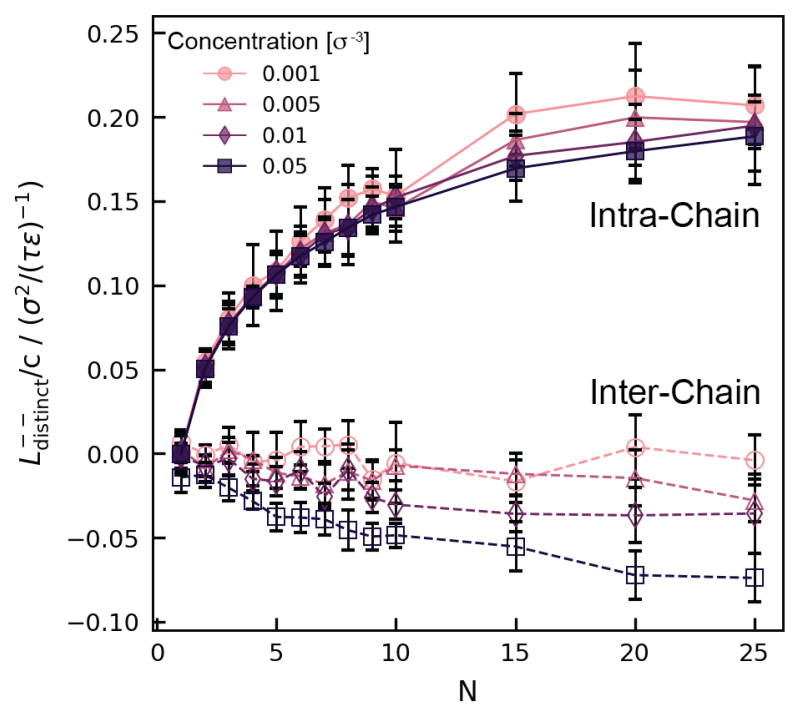

Figure S5: $L_{\text {distinct }}^{--} / c$ split into contributions from anion-anion correlations within chains (solid lines, filled markers) and between chains (dashed lines, open markers). The two curves sum to yield the total $L_{\text {distinct }}^{--} / c$.

\section{Stefan-Maxwell Coefficients}

The Stefan-Maxwell equations for multicomponent diffusion may be written as

$$
c_{i} \nabla \bar{\mu}_{i}=\sum_{j \neq i} K^{i j}\left(\boldsymbol{v}_{j}-\boldsymbol{v}_{i}\right)
$$

where $K^{i j}$ are the Stefan-Maxwell coefficients and the other quantities are defined in the main text. For a binary electrolyte, the Onsager transport coefficients $L^{i j}$ may be mapped to $K^{i j}$ via the following relations, derived in Fong et al.: ${ }^{\text {S1 }}$

$$
\begin{aligned}
& K^{+-}=\omega_{+} \omega_{-} \frac{\hat{L}^{00}+\hat{L}^{+-}-\hat{L}^{+0}-\hat{L}^{-0}}{\hat{L}^{+0} \hat{L}^{-0}-\hat{L}^{+-} \hat{L}^{00}}, \\
& K^{+0}=\omega_{+} \omega_{0} \frac{\hat{L}^{--}+\hat{L}^{+0}-\hat{L}^{+-}-\hat{L}^{-0}}{\hat{L}^{+-} \hat{L}^{-0}-\hat{L}^{+0} \hat{L}^{--}}, \\
& K^{-0}=\omega_{-} \omega_{0} \frac{\hat{L}^{++}+\hat{L}^{-0}-\hat{L}^{+-}-\hat{L}^{+0}}{\hat{L}^{+-} \hat{L}^{+0}-\hat{L}^{-0} \hat{L}^{++}},
\end{aligned}
$$


where $\hat{L}^{i k}=\frac{L^{i k}}{c_{i} c_{k}}, \omega_{i}$ is the mass fraction of species $i$, and the solvent transport coefficients may be obtained from $L^{++}, L^{+-}$, and $L^{--}$using the constraint $\sum_{i} M_{i} L^{i j}=0$. The StefanMaxwell coefficients obtained from the polyelectrolyte solutions in this work are given in Figure S6.
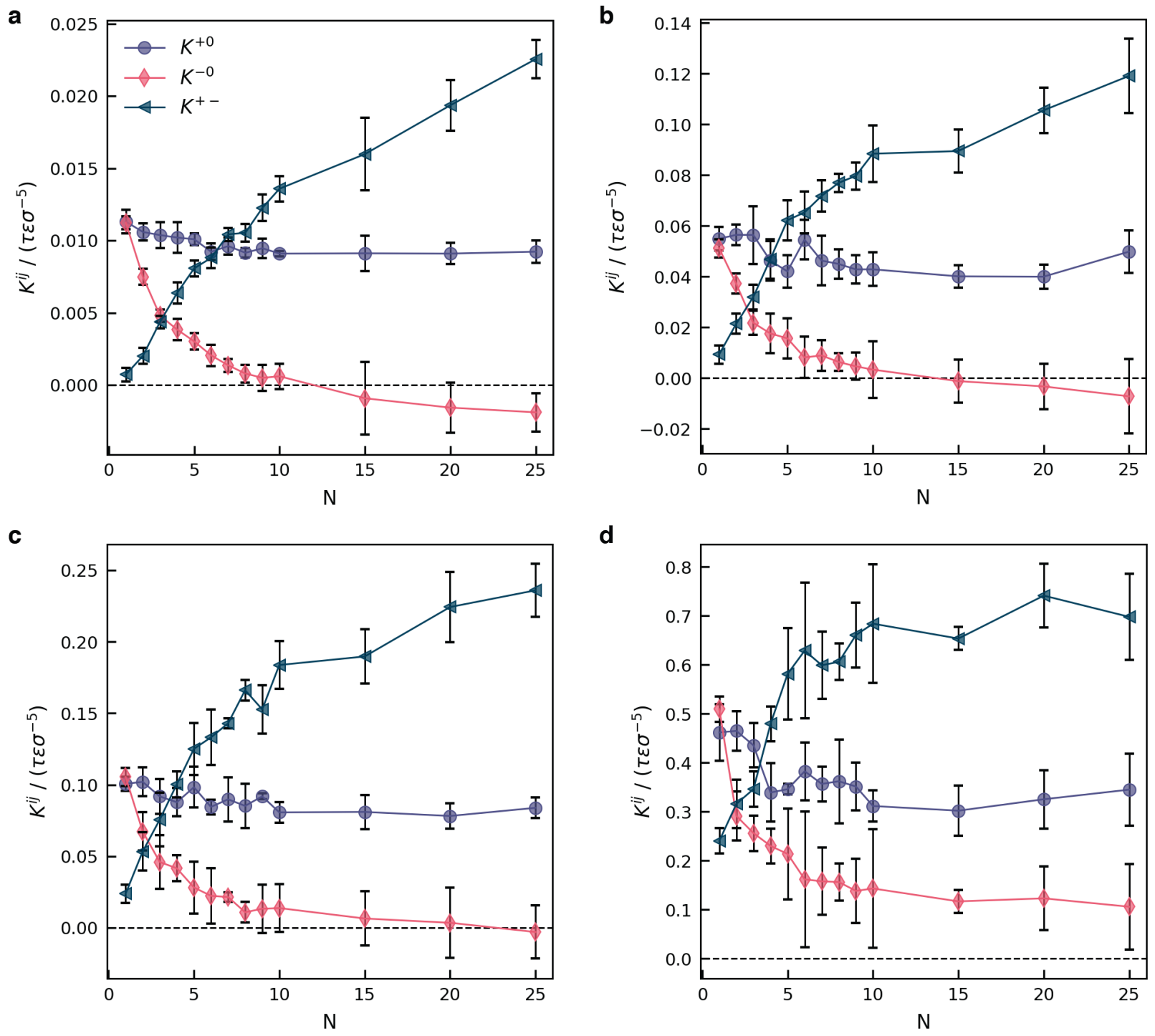

Figure S6: Stefan-Maxwell coefficients $K^{i j}$ for concentrations of (a) 0.001, (b) 0.005, (c) 0.01 , and (d) $0.05 \sigma^{-3}$. Note that in some cases $K^{-0}$ transitions from positive to negative, corresponding to divergence of the Stefan-Maxwell diffusion coefficients $D^{i j}$, where $K^{i j}=$ $\frac{R T c_{i} c_{j}}{c_{\mathrm{T}} D^{i j}}$. 


\section{Experimentally-measurable transport properties}
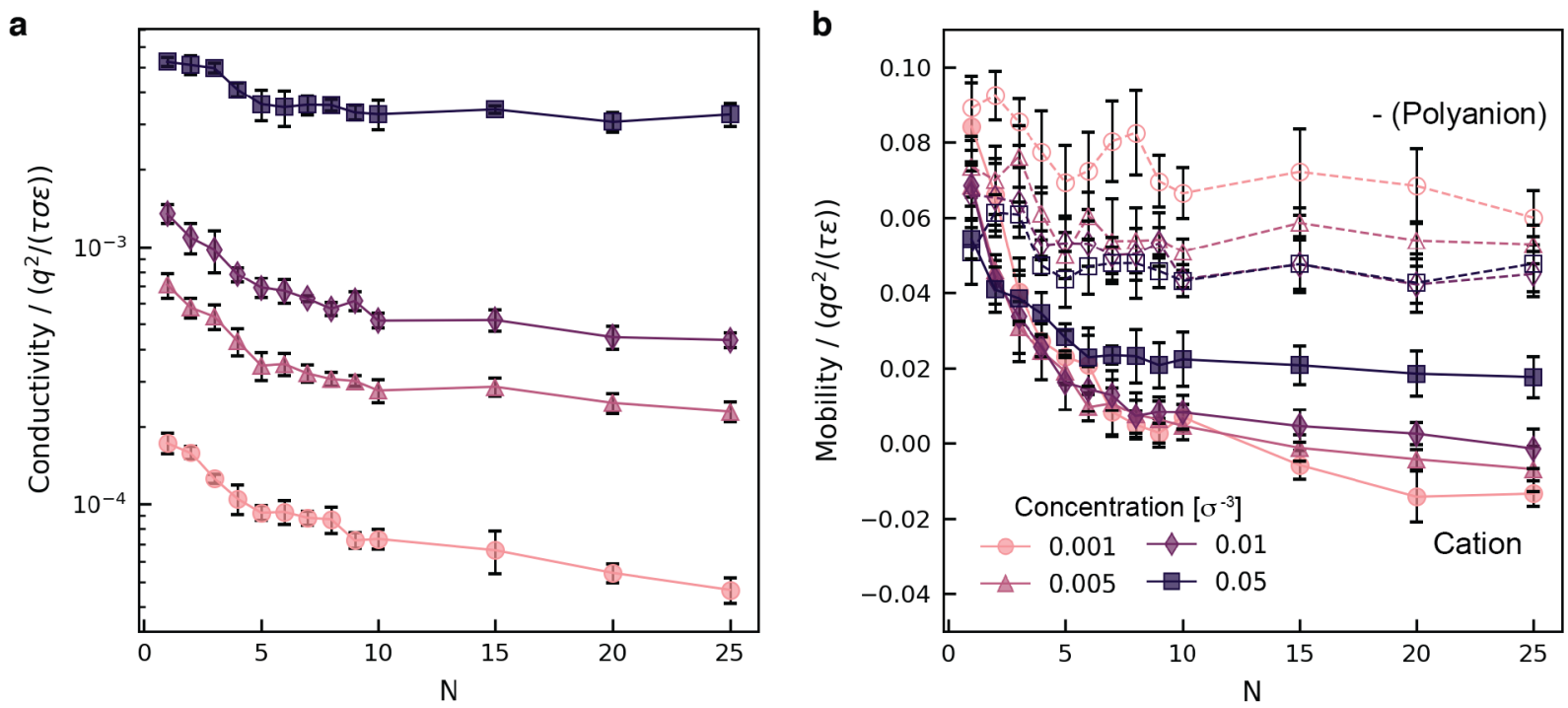

Figure S7: Additional experimentally-relevant quantities computed from the transport coefficients $L^{i j}$. (a) Ionic conductivity, $\kappa$. (b) Electrophoretic mobility for the cation (solid lines) and polymer (dashed lines). As the polymer mobility is negative, $-u_{\text {polymer }}$ is shown.
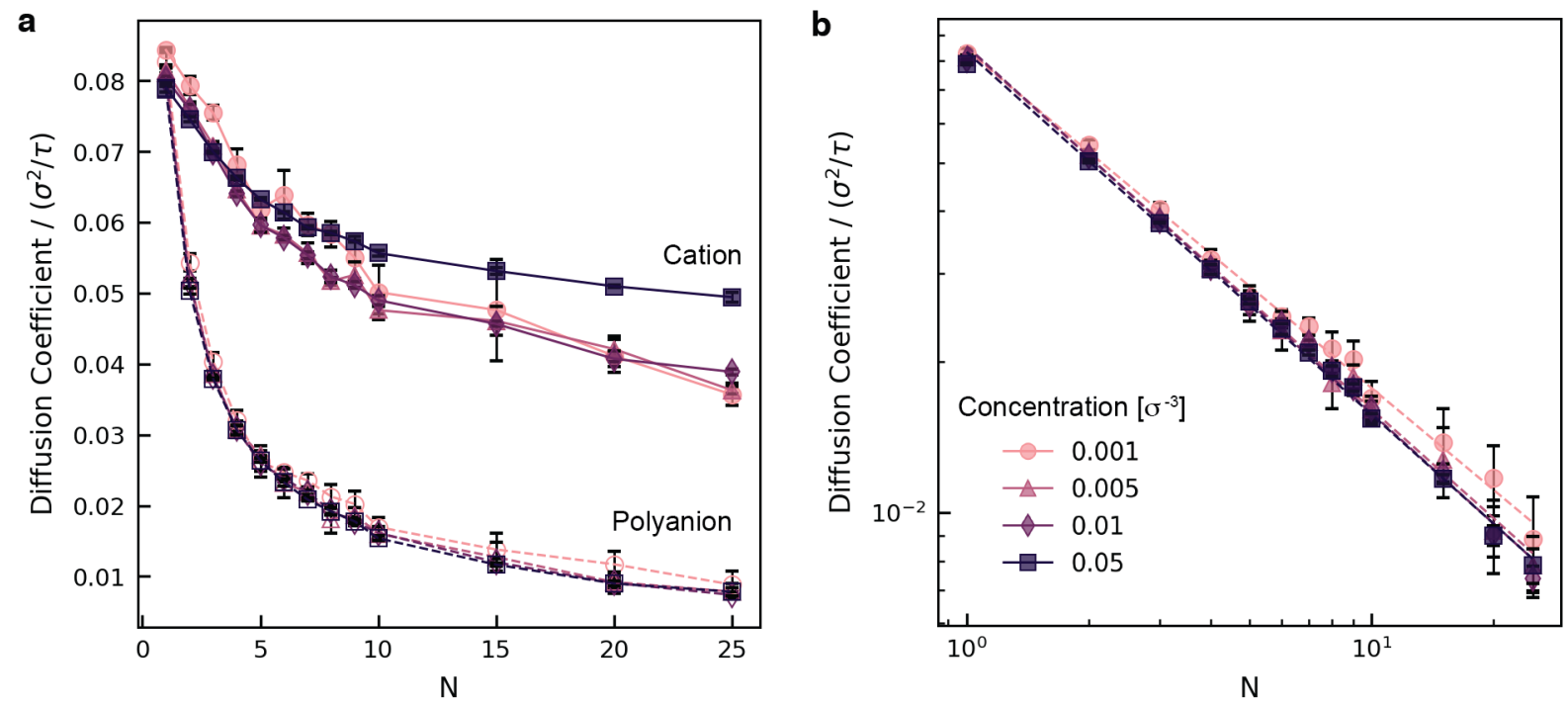

Figure S8: Diffusion coefficients. (a) Diffusion coefficients for both the cation (solid lines) and polymer (dashed lines) as a function of chain length. Note that we have sampled sufficiently long times such that the diffusion coefficient of the polymer center of mass is equal to that of individual monomers. (b) Polymer diffusion coefficient on a log-log scale. Dashed lines show fits of the form $D_{\text {polymer }} \sim N^{-m}$, where $m$ is a fitting parameter given for each concentration in Table S1. 
Table S1: Scaling exponents $m$ for polymer diffusion as a function of chain length, $D_{\text {polymer }} \sim$ $N^{-m}$.

\begin{tabular}{|l||cccc|}
\hline Concentration $\left(\sigma^{-3}\right)$ & 0.001 & 0.005 & 0.01 & 0.05 \\
\hline Scaling Exponent $m$ & 0.68 & 0.72 & 0.73 & 0.73 \\
\hline
\end{tabular}

\section{Solvent-Free System}
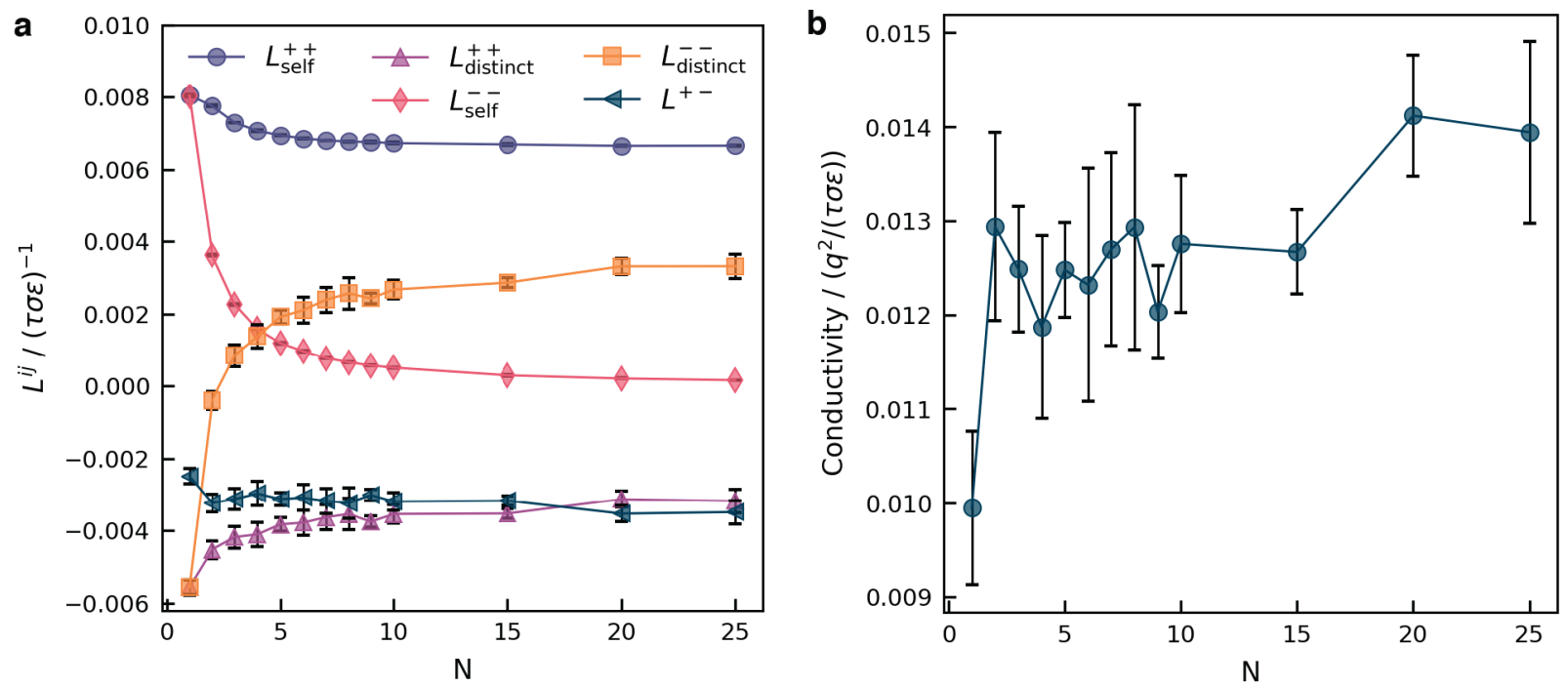

Figure S9: (a) Transport coefficients for the solvent-free system as a function of chain length, split into self and distinct terms. (b) Conductivity as a function of chain length for the solvent-free system. 


\section{Polymer structure and dynamics}
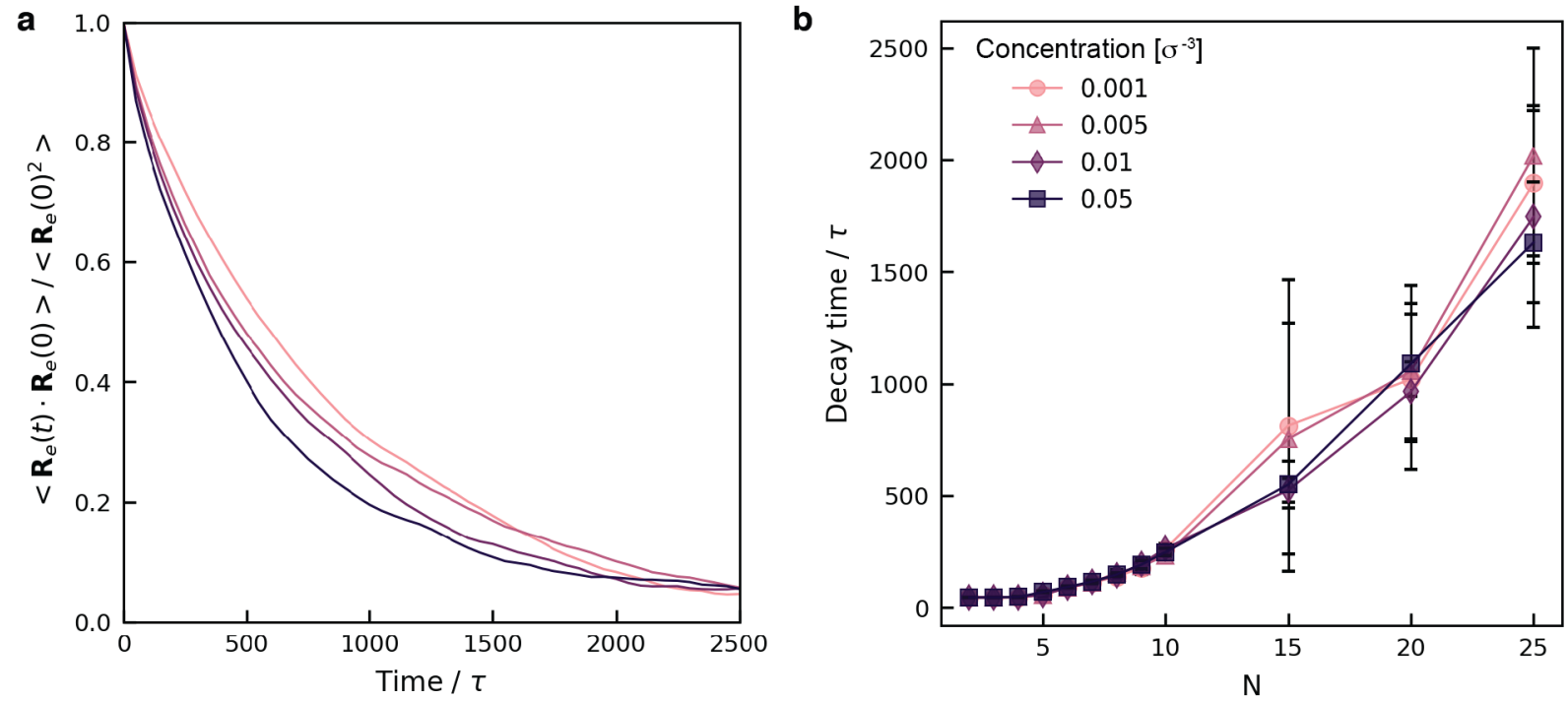

Figure S10: End-to-end vector analysis. (a) End-to-end vector autocorrelation functions for $N=25$ at each concentration. (b) Decay times of the end-to-end vector autocorrelation functions (defined as the time to reach a value of 0.1 ).

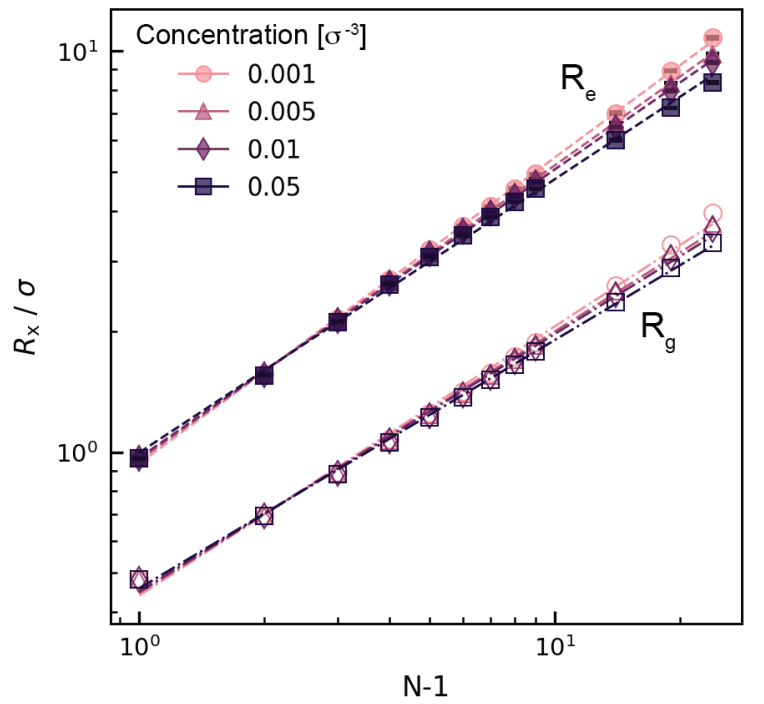

Figure S11: Polymer end-to-end distance $\left(R_{\mathrm{e}}\right.$, closed symbols $)$ and radius of gyration $\left(R_{\mathrm{g}}\right.$, open markers) as a function of chain length for each concentration. Dashed lines show fits to the equation $R_{\mathrm{x}} \sim(N-1)^{\nu}$, where $\nu$ is a fitting parameter given for each concentration in Table S2. 
Table S2: Scaling exponents $\nu$ for polymer end-to-end distance and radius of gyration as a function of chain length, $R_{\mathrm{x}} \sim(N-1)^{\nu}$.

\begin{tabular}{|c||cccc|}
\hline Concentration $\left(\sigma^{-3}\right)$ & 0.001 & 0.005 & 0.01 & 0.05 \\
\hline$R_{\mathrm{e}}$ Scaling Exponent $\nu$ & 0.76 & 0.73 & 0.72 & 0.68 \\
\hline$R_{\mathrm{g}}$ Scaling Exponent $\nu$ & 0.67 & 0.65 & 0.64 & 0.62 \\
\hline
\end{tabular}

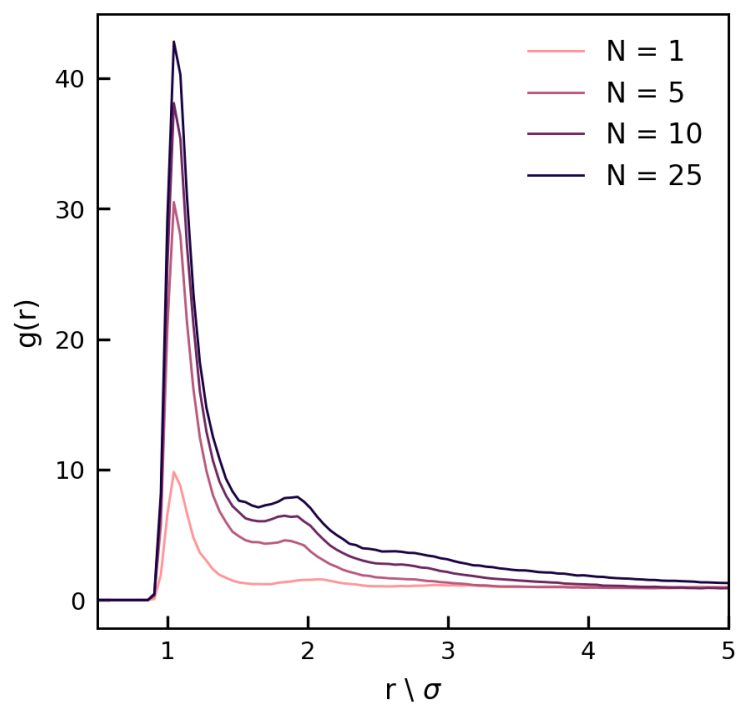

Figure S12: Representative cation-anion radial distribution functions $(g(r))$ at a concentration of $0.01 \sigma^{-3}$ for four different chain lengths. 


\section{References}

(S1) Fong, K. D.; Bergstrom, H. K.; McCloskey, B. D.; Mandadapu, K. K. Transport phenomena in electrolyte solutions: Non-equilibrium thermodynamics and statistical mechanics. arXiv preprint arXiv:2006.16164 2020, 